\title{
Rigid-body kinematics Versus Flapping Kinematics of a Flapping Wing Micro Air Vehicle
}

\author{
J. V. Caetano* \\ Portuguese Air Force Academy Research Laboratory, Sintra, Portugal

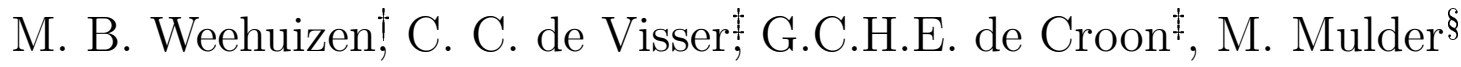 \\ Control and Simulation Department, Aerospace Faculty, Delft University of Technology, \\ Delft, The Netherlands
}

Several formulations have been proposed to model the dynamics of ornithopters, with inconclusive results regarding the need for complex kinematic formulations. Furthermore, the impact of assumptions made in the collected results was never assessed by comparing simulations with real flight data. In this study two dynamic models of a Flapping Wing Micro Aerial Vehicle (FWMAV) were derived and compared: a) single rigid body aircraft equations of motion and b) Virtual Work Principle derivation for multiple rigid body flapping kinematics. The aerodynamic forces and moments were compared by feeding the states that were reconstructed from the position and attitude data of a 17 gram free flying FWMAV into the dynamic equations of both formulations. To understand the applicability of rigid body formulations to FWMAVs, six wing-to-body mass ratios and two wing configurations were studied using real flight data. The results show that rigid body models are valid for the aerodynamic reconstruction of FWMAVs with four wings in ' $\mathrm{X}$ ' configuration and two-winged FWMAV

*Ph.D. candidate, Section of Control and Simulation, Aerospace Faculty, Delft University of Technology; AIAA Member.

${ }^{\dagger}$ Ms.C., Section of Control and Simulation, Aerospace Faculty, Delft University of Technology

$\ddagger$ Assistant Professor, Section of Control and Simulation, Aerospace Faculty, Delft University of Technology; AIAA Member

$\S$ Professor, Section of Control and Simulation, Aerospace Faculty, Delft University of Technology; AIAA Member 
Final Manuscript available on JOURNAL OF GUIDANCE, CONTROL, AND DYNAMICS

DOI 10.2514/1.G000923

http://arc.aiaa.org/doi/abs/10.2514/1.G000923

with a total wing-to-body mass ratio below $24 \%$ and $5.6 \%$, respectively, without considerable information loss.

\section{Nomenclature}

\section{Acronyms}

$\mathrm{CG}$

Center of Gravity

EOM Equations of Motion

FWMAV Flapping Wing Micro Air Vehicle

\section{Variables}

$(p, q, r) \quad$ Angular Velocities in the body frame axes

$(\dot{p}, \dot{q}, \dot{r}) \quad$ Angular Accelerations in the body frame axes

$(u, v, w) \quad$ Linear velocities in the body frame axes

$(\dot{u}, \dot{v}, \dot{w}) \quad$ Linear Accelerations in the body frame axes

$(L, M, N)$ Aerodynamic Moments in the body axes

$(X, Y, Z) \quad$ Aerodynamic Forces in the body axes

$C_{\theta_{w_{0}}} \quad$ Affine coefficient for estimated wing pitch angle

$C_{\theta_{w_{\zeta}}} \quad$ Coefficient for $\zeta$

$C_{\theta_{w_{\dot{\zeta}}}} \quad$ Coefficient for $\dot{\zeta}$

$I_{i} \quad$ Moment of Inertia of the ornithopter in the $i$ axis

$I_{i j} \quad$ Product of Inertia of the ornithopter in the $i j$ product axes

$N_{i} \quad$ Total number of state observations

$Q_{j} \quad$ Generalized forces

$R^{2} \quad$ Coefficient of Determination

T Flap period

$\boldsymbol{F}_{\boldsymbol{i}} \quad$ External acting forces

$\boldsymbol{M}_{\boldsymbol{g}} \quad$ Moment caused by gravity force in the Body frame

$\boldsymbol{M}_{\boldsymbol{i}} \quad$ External acting moments

$\boldsymbol{q}_{j} \quad$ Array of generalized coordinates

$\boldsymbol{u}_{j} \quad$ Array of quasi-velocities

$\boldsymbol{v}_{i} \quad$ Linear velocity of body $i$ in the Body frame

$\dot{\boldsymbol{v}}_{i} \quad$ Linear acceleration of body $i$ in the Body frame

$\mathcal{R}_{b, I} \quad$ Rotation matrix from Inertial to Body frame, in the Bing frame

$\mathcal{R}_{b, w_{i}} \quad$ Rotation matrix from Body to Wing frame $i$, in the Body frame

$\mathcal{R}_{w 1, b} \quad$ Rotation matrix from Body to Wing frame $i$, in the Wing frame

$m_{i} \quad$ Mass of body $i$

$n \quad$ Total number of bodies in flapping model 
Final Manuscript available on JOURNAL OF GUIDANCE, CONTROL, AND DYNAMICS

DOI 10.2514/1.G000923

http://arc.aiaa.org/doi/abs/10.2514/1.G000923

$t \quad$ Time instant

$t^{*} \quad$ Dimensionless time with respect to flap period

$\left(e_{0}, e_{1}, e_{2}, e_{3}\right)$ Quaternion vector

\section{Greek}

$(\phi, \theta, \psi) \quad$ Euler Angles - Roll, Pitch, Yaw

$(\dot{\phi}, \dot{\theta}, \dot{\psi}) \quad$ Attitude Angle rates

RMSE Root Mean Square of the Error

$\boldsymbol{\beta}_{\boldsymbol{i j}} \quad$ Angular velocity coefficient matrix

$\delta_{f} \quad$ Flapping frequency input

$\delta_{e} \quad$ Elevator angle input

$\delta_{r} \quad$ Rudder angle input

$\gamma_{i j} \quad$ Linear velocity coefficient matrix

$\boldsymbol{g}_{\boldsymbol{b}} \quad$ Gravity vector in the Body frame

$\boldsymbol{\omega}_{i} \quad$ Angular velocity of body $i$ in the Body frame

$\dot{\boldsymbol{\omega}}_{i} \quad$ Angular velocity of body $i$ in the Body frame

$\boldsymbol{\rho}_{b_{w i, h}} \quad$ Vector from CG of wing $i$ to hinge point, in the Body frame

$\boldsymbol{\rho}_{c i} \quad$ Vector from the reference point of a body to its center of mass

$\ddot{\boldsymbol{\rho}}_{c i} \quad$ Acceleration of vector $\boldsymbol{\rho}_{c i}$ in the Body frame

$\boldsymbol{\rho}_{h, b} \quad$ Vector from wing hinge point to CG of the main body

$\boldsymbol{\rho}_{w_{i}, h} \quad$ Vector from CG of wing $i$ to hinge point, in the Wing frame

$\theta_{w_{i}} \quad$ Pitch angle of the wing using chord reference line $i$

$\hat{\theta}_{w_{i}} \quad$ Estimated wing pitch angle using chord reference line $i$

$\zeta_{0} \quad$ Dihedral of the wings

$\zeta \quad$ Wing angle

$\dot{\zeta} \quad$ Flap angular velocity

$\ddot{\zeta} \quad$ Flap angular acceleration

\section{Reference Frames and Subscripts}

$B \quad$ Body Reference Frame

I Inertial Frame Subscript

I Inertial Reference Frame

$W_{i} \quad$ Reference Frame of wing $i$

$b \quad$ Body Frame Subscript

$w \quad$ Wing Frame Subscript

$\left(\boldsymbol{x}_{\boldsymbol{I}}, \boldsymbol{y}_{\boldsymbol{I}}, \boldsymbol{z}_{\boldsymbol{I}}\right)$ Unit axes of the Body reference frame

$\left(\boldsymbol{x}_{\boldsymbol{b}}, \boldsymbol{y}_{\boldsymbol{b}}, \boldsymbol{z}_{\boldsymbol{b}}\right)$ Unit axes of the Body reference frame

$\left(\boldsymbol{x}_{\boldsymbol{w}}, \boldsymbol{y}_{\boldsymbol{w}}, \boldsymbol{z}_{\boldsymbol{w}}\right)$ Unit axes of the Wing reference frame 
Final Manuscript available on JOURNAL OF GUIDANCE, CONTROL, AND DYNAMICS

DOI 10.2514/1.G000923

http://arc.aiaa.org/doi/abs/10.2514/1.G000923

\section{Introduction}

The evolution of Flapping Wing Micro Aerial Vehicles (FWMAVs) has lead to the development of high-end bioinspired robots, such as Aerovironment's Nano-Hummingbird [1], Harvard's RoboBee [2], Carnegie Mellon's [3] and UC Berkeley's [4] FWMAVs and TU Delft's DelFlys [5]. These benefit from having a more extensive flight envelope than conventional fixed or rotary wing platforms, as they are capable of hover, fast forward flight and perching, while making use of efficient lift generation strategies when in forward flight.

To take full advantage of the capabilities of the FWMAV, an accurate dynamic model is needed for automatic control and design improvement. Such model can either relate the inputs and states to the outputs through black-box or nonlinear dynamic inversion approaches, or it can use a white-box phenomenological approach applied to a set of consecutive kinematic and aerodynamic blocks. In the latter approach, the kinematic block is the basis of the dynamic models, which will affect the aerodynamic reconstruction and aerodynamic identification cycle, as presented in Fig. 1.

There is no consensus on how representative rigid body kinematic models are of flapping wing systems, with several studies arriving at contradicting results. While some authors claim the need for devising complex equations of motion that model the flapping and have used, e.g., Newton-Euler's force principles [6], Kane's equations [7], Lagrange's energy-based methods [8] or d'Alembert's virtual work principle [9, 10], other authors [11, 12] have used simple aircraft equations of motion to simulate the behavior of bird-like FWMAV and have demonstrated dynamic model stability, as well as a flying simulation.

Flapping wing kinematic formulations can be complicated to derive [7, 8, 10] and are not practical for iterative system development, as new formulations have to be devised for each change in the design of the FWMAV. This way, rigid body kinematic models would facilitate system identification techniques and the derivation of phenomenological aerodynamic models that could more easily be used for onboard flight control strategies and simulations.

Only a few studies have used real flying robots for aerodynamic characterization [8, 13, and none of them focused on comparing the impact of the kinematic formulations in system identification or force simulation. A first comparison between two dynamic formulations that represented a real FWMAV was attempted by [14], but this work only included one degree of freedom (d.o.f.) on the wings and its results only addressed the applicability of each formulation to that particular FWMAV.

The current study aims at better understanding the need for complex kinematic formulations for the aerodynamic identification of FWMAVs by assessing the applicability of rigid body kinematics to bioinspired FWMAV. It is applicable to ornithopters inspired in bird species like the Pomatorhinus Ruficollis or the Coturnix Coturnix [15] or in insect 
species like the Schistocerca Gregaria [16] or the Manduca Sexta [17], in terms of body mass, wing-to-body mass ratio and wing flap frequency, as well as to ornithopters with ' $\mathrm{X}$ ' wing configuration, used in Academia [18, 19] and industry.

The study is done through the comparison of the aerodynamic forces and moments reconstructed from real flight data using two different dynamic models: a) simple Newton Euler's single rigid body general aircraft equations of motion and b) complex Virtual Work Principle multi-rigid body flapping formulation. The analyses are further extended by changing the wing-to-body mass ratios of simulated FWMAVs using the same flight data to predict the effects of wing mass and number of wings on the reconstructed aerodynamic forces and moments. This provides a wing-to-body mass ratio confidence interval for the application of rigid body kinematics to FWMAVs with four and two wings that have a similar configuration to the ornithopter that was used in the flight tests. A description of the present study and its involving framework is presented in Fig. 1.

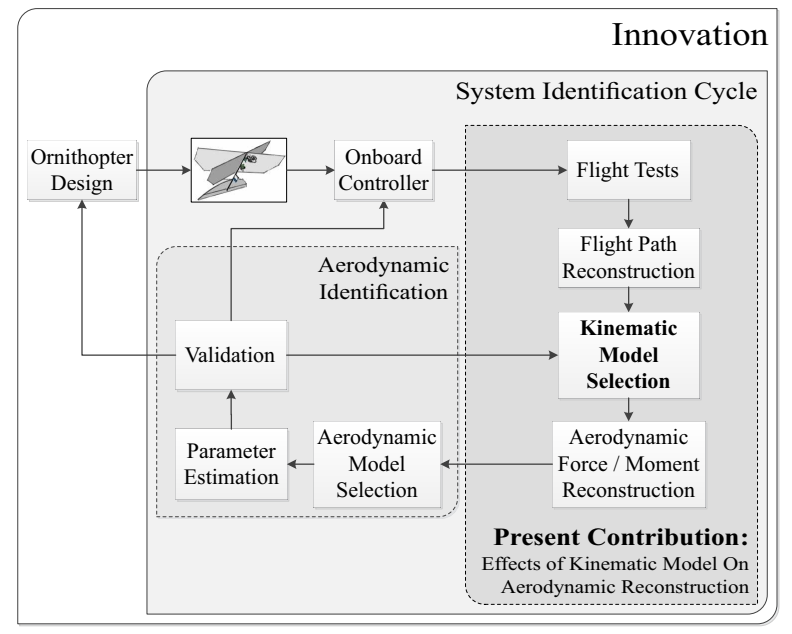

Figure 1. Contribution of the current study in the System Design and Identification cycles.

The article is structured as follows: it starts by presenting the FWMAV model in Section III and continues with the derivation of two dynamic formulations. Section III presents the experimental techniques and the states reconstructed from the free flight tests. In Section IV the aerodynamic forces and moments were calculated for the real FWMAV and used to assess the applicability of rigid body kinematics to different FWMAVs. Section $\square$ concludes that rigid body formulations are applicable to FWMAVs within a certain wing-to-body mass ratio.

\section{Model and Dynamic Formulations}

This section presents the mathematical principles and assumptions of the two dynamic formulations that are compared in this study. It starts by presenting the FWMAV model, 
Final Manuscript available on JOURNAL OF GUIDANCE, CONTROL, AND DYNAMICS

DOI 10.2514/1.G000923

http://arc.aiaa.org/doi/abs/10.2514/1.G000923

followed by the two dynamic formulations that were derived for that model.

The formulations have different approaches. The first, typically known as the general aircraft equations of motion, uses Newton-Euler's principle to derive the equations of motion of a rigid non-flapping body. The second uses the Principle of Virtual Work applied to rigid bodies to determine the kinematics of a flapping platform.

\section{A. The Flapping Wing Micro Aerial Vehicle Model}

The FWMAV used for the present study, named DelFly II, has four wings in ' $\mathrm{X}$ ' configuration and an inverted ' $\mathrm{T}$ ' tail (Figs. 2 and 3). Its wings are made of Mylar foil and very thin carbon rods, having a closed wing dihedral of $13^{\circ}$. This wing configuration minimizes the flapping-induced oscillations in the body due to the opposite movement of the upper and lower wings, which rotate with symmetric angular velocities and accelerations around $\boldsymbol{x}_{\boldsymbol{b}}$ (Fig. 2).

The tail of the ornithopter is made from thin Styrofoam for rapid construction, robustness and visual referencing in flight. It introduces static stability on the platform and can be used for attitude control through its elevator and rudder. It also simplifies the wing design and control strategies by decoupling the lateral control from the wings, thus allowing it to have two d.o.f. (active rotation around $\boldsymbol{x}_{\boldsymbol{b}}$ and passive rotation around the leading edge spar) with only one control input.

This ornithopter is capable of sustained flight from hover to $7 \mathrm{~m} / \mathrm{s}$ of total flight speed. Depending on the payload, it flaps at frequencies between 12 and $14 \mathrm{~Hz}$ for regimes close to hover, reducing the flap rate down to $8 \mathrm{~Hz}$ for fast forward flight.

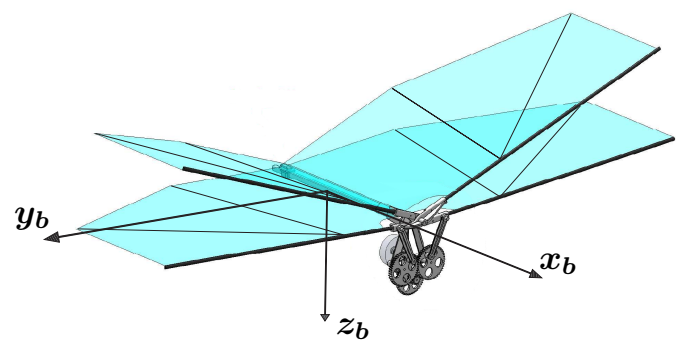

Figure 2. Wing configuration and Body frame axis $\left(x_{b}, y_{b}, z_{b}\right)$.

Table 1 presents the masses of the composing parts of the DelFly. These will be mentioned further in the article to explain some of the assumptions that were taken. 
Final Manuscript available on JOURNAL OF GUIDANCE, CONTROL, AND DYNAMICS

DOI 10.2514/1.G000923

http://arc.aiaa.org/doi/abs/10.2514/1.G000923

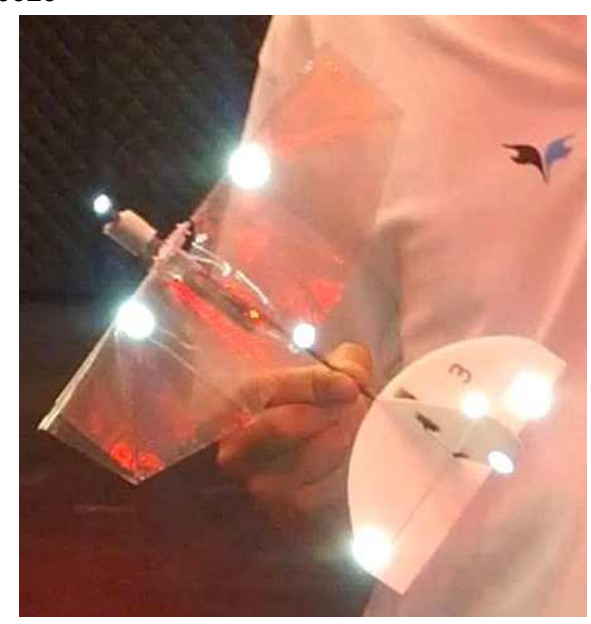

Figure 3. The DelFly FWMAV with reflective markers used in the flight testing.

\section{B. Single Rigid Body Dynamic Model}

\section{Model and Reference Frames}

The first model approach considers the DelFly as a rigid body with no moving parts. Six assumptions were taken in this rigid body kinematic formulation: a) non-flapping rigid body; b) constant mass; c) no inertia changes due to flapping or bending; d) symmetric platform; e) stationary atmosphere; f) flat Earth.

The Body reference frame is attached to the main body, with its origin at the CG. The main body is aligned with the $\boldsymbol{x}_{\boldsymbol{b}}$ axis; the $\boldsymbol{y}_{\boldsymbol{b}}$ axis points out of the right side of the DelFly; the $\boldsymbol{z}_{b}$ points down, perpendicular to the $x_{b} y_{b}$ plane, as indicated in Fig. 2. 
Final Manuscript available on JOURNAL OF GUIDANCE, CONTROL, AND DYNAMICS

http://arc.aiaa.org/doi/abs/10.2514/1.G000923

Table 1. Masses of the Components of the Ornithopter

\begin{tabular}{lc}
\hline Component & Mass (g) \\
\hline Main Rod & 0.791 \\
Autopilot & 1.454 \\
Battery & 5.640 \\
Servo (x2) & 0.536 \\
Driving Mechanism & 3.182 \\
Horizontal Tail Structure & 1.215 \\
Vertical Tail Structure & 0.279 \\
Reflector balls (x3) & 0.33 \\
Other (glue + wires) & 1.48 \\
Body \& Tail & 16.2 \\
\hline Wing & 0.298 \\
\hline Total & 17.4 \\
\hline Wing-to-Body Mass ratio & $1.8 \%$ \\
\hline
\end{tabular}

\section{Mathematical Formulation}

The Newton-Euler based rigid body equations of the DelFly are given by [20]:

$$
\begin{aligned}
& X=m(g \sin \theta+\dot{u}+q w-r v) \\
& Y=m(-g \sin \phi \cos \theta+\dot{v}+r u-p w) \\
& Z=m(-g \cos \phi \cos \theta+\dot{w}+p v-q u) \\
& L=I_{x} \dot{p}-I_{x z}(\dot{r}+p q)+\left(I_{z}-I_{y}\right) q r \\
& M=I_{y} \dot{q}+\left(I_{x}-I z\right) r p+I_{x z}\left(p^{2}-r^{2}\right) \\
& N=I_{z} \dot{r}-I_{x z} \dot{p}+\left(I_{y}-I_{x}\right) p q+I_{x z} r q \\
& \dot{\psi}=(q \sin \phi+r \cos \phi) / \cos \theta \\
& \dot{\theta}=q \cos \theta-r \sin \theta \\
& \dot{\phi}=p+(q \sin \phi+r \cos \phi) \tan \theta
\end{aligned}
$$

The first six equations represent the acting aerodynamic forces $(X, Y, Z)$ and moments $(L, M, N)$ along the body axes of the vehicle $\left(\boldsymbol{x}_{\boldsymbol{b}}, \boldsymbol{y}_{\boldsymbol{b}}, \boldsymbol{z}_{\boldsymbol{b}}\right)$. The translational and angular velocities are represented by $(u, v, w)$ and $(p, q, r)$, respectively, while $(\dot{u}, \dot{v}, \dot{w})$ are the linear accelerations at the $\mathrm{CG}$ and the last three equalities relate the Euler angles $(\phi, \theta, \psi)$ and 
Final Manuscript available on JOURNAL OF GUIDANCE, CONTROL, AND DYNAMICS

DOI 10.2514/1.G000923

http://arc.aiaa.org/doi/abs/10.2514/1.G000923

rates $(\dot{\phi}, \dot{\theta}, \dot{\psi})$ to the body angular velocities.

\section{Applicability Aspects}

The previous formulation does not account for any moving parts and considers the inertia tensor as being constant. Moreover, the non-flapping validity region depends not only on the wing-generated evolution of the forces and moments over the flap cycle but also on the wing mass to body ratio and the wing inertia to body inertia ratio.

The rigid body formulation, however, benefits from being easily devised for non-exotic aircraft configurations while still taking into consideration the accelerations and Coriolis couplings of rigid body dynamics. This approach is justified by the wing configuration of the ornithopter that minimizes the CG oscillations over a flap cycle, as the lower wing flap counteracts the upper wing's to a great extent, as shown in Figure 4 .

Furthermore, Figure 4 compares the position of CG of the DelFly with a similar ornithopter that would have only two wings similar to the ones on the test ornithopter. It is known [5] that the ' $\mathrm{X}$ ' wing configuration maximizes lift and thrust production by more that $150 \%$ when compared to a two-winged ornithopter. Hence, this comparison is still conservative, since a two-winged ornithopter would need bigger and heavier wings for sustained flight thus inducing bigger oscillations on the CG location.
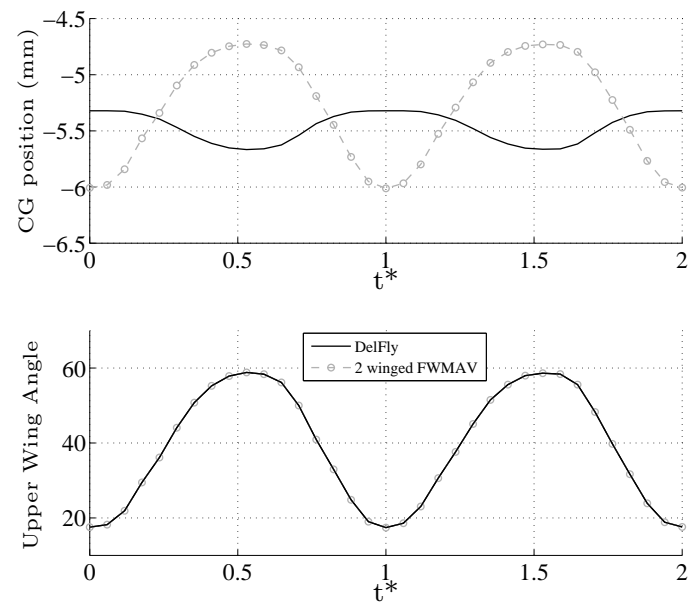

Figure 4. Location of the CG with respect to the body carbon rod within two flapping cycles for the DelFly and for a two-winged ornithopter with similar wings.

The DelFly is manually built making it not perfectly symmetric around the $x_{b} z_{b}$ plane. However, the symmetry assumption is justified by the small moments of inertia around $\boldsymbol{x}_{\boldsymbol{b}}$ and crossed moments of inertia, making the coupled $I_{x y}, I_{y z}$ negligible, here assumed to be zero. 
Final Manuscript available on JOURNAL OF GUIDANCE, CONTROL, AND DYNAMICS

DOI 10.2514/1.G000923

http://arc.aiaa.org/doi/abs/10.2514/1.G000923

The last three equalities of Eq. 1 are not well defined for pitch angles close to $90^{\circ}$ since a small variation in yaw will induce a large oscillation in $\psi$, resulting in inadequate information in $\dot{\psi}$ and consequently wrong angular velocity $r$ determination. The same happens to the roll angle, as a rotation around $\boldsymbol{z}_{\boldsymbol{b}}$ affects the heading angle $\psi$ which, in turn, feeds a rolling miss-information to the Euler angles.

Moreover, the singularity at $\theta=90^{\circ}$ denies the calculation the Euler angles, and hence, the rotational rates $(p, q, r)$. The ornithopter can enter such singularity conditions for certain elevator pitch-up maneuvers. Thus, to prevent $p, q, r$ from feeding wrong information into the force and moments equations, new attitude angles were devised. This methodology is explained in [21].

\section{Multi-Rigid Flapping Wing Dynamic Model}

\section{Model and Reference Frames}

The second method models the DelFly as a combination of five rigid bodies - a main body and four wings. The main body includes the carbon rod that connects the parts, the hardware attached to the rod, as well as the vertical and horizontal tails. In the real ornithopter, only the elevator and rudder have one degree of freedom with respect to the rigid body. These surfaces vibrate during flight due to the flapping, given the play that the small servos allow - as can be seen in Fig. 10. However, their low mass (0.46g for the elevator and $0.15 \mathrm{~g}$ for he rudder) compared to the total mass of the body (16.2g) associated with the small amplitudes of vibration make their contribution to the kinematics nearly non-existent, which justified the modeling of the components as a single rigid body.

The wing bodies, identified as $W_{1}, W_{2}, W_{3}$ and $W_{4}$ in Fig. 6b, on the contrary, are modeled separately since all of them have different kinematics that will affect the overall kinematic behavior of the ornithopter. The Body reference frame (B) was defined as in the previous rigid body method, and is presented in Fig. 2. The reference frames and $\theta_{w}$ are depicted in Fig. 5

Wing Frame (W) A separate reference frame was used for each wing, whose origin is at the hinge point and the $x_{w} y_{w}$ plane coincident with the wing surface. The frames are assumed to rotate with the wing around $\boldsymbol{x}_{\boldsymbol{b}}$ and $\boldsymbol{y}_{\boldsymbol{w}}$.

Two vectors describe the motion of the CG of all wings in the Body frame: $\boldsymbol{\rho}_{h, b}$ represents the position of the hinge point with respect to the CG of the main body; $\boldsymbol{\rho}_{w, h}$ connects the hinge point to the $\mathrm{CG}$ of the wings, as indicated in Fig. 6a.

Wing Modeling The four wings are modeled as rigid bodies that are geometrically identical to the real wings [14]. The wing models have two d.o.f.: active rotation around $\boldsymbol{x}_{\boldsymbol{b}}$ with 


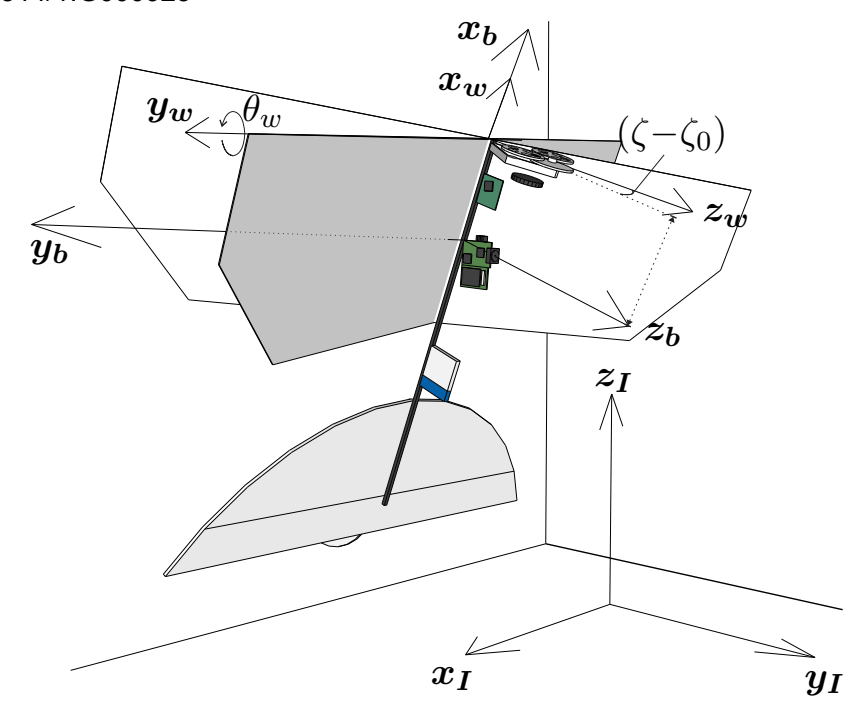

Figure 5. Inertial $\left(x_{I}, y_{I}, z_{I}\right)$, Body $\left(x_{b}, y_{b}, z_{b}\right)$ and Wing $\left(x_{w}, y_{w}, z_{w}\right)$ reference frames.

an angle $\zeta$ (Fig. 6b) and passive rotation around $\boldsymbol{y}_{\boldsymbol{w}}$ with an angle $\theta_{w}$ (Fig 5 ). The wings are set at a dihedral angle $\zeta_{0}$ of $13^{\circ}$ and the flapping angle $\zeta$ is defined from $\zeta_{0}$ to the wing for all wings, as shown in Fig. 6b.

For the current model, the wing pitch angle $\left(\theta_{w}\right)$ is taken around the $\boldsymbol{y}_{\boldsymbol{w}}$ axis of the wing as being the angle between the chord line of the wing foil and the $x_{b} y_{b}$ plane. This angle will determine the evolution of the CG of the wing during the flap cycle. Figure 7 presents the chordwise evolution of the lower wing surface of the real ornithopter during one flap cycle for a section located at $70 \%$ of the wing span. It was processed from high speed imagery collected when testing the DelFly in a wind tunnel [22]. It is divided in six time-steps: first three subfigures (a, b, c) represent the out-stroke motion; last three subfigures (d, e, f) represent in-stroke motion; $t^{*}=\frac{t}{T}$ represents dimensionless time, with $T$ being the period of the flap. Bottom wings are assumed to have a mirrored evolution.

It is important to use the real wing pitch angle information in the kinematic modeling. In order to add one d.o.f. to the model (wing pitch angle) and keep the same number of generalized coordinates, the wing pitch angle $\theta_{w}$ of the model has to be a function of the wing flap angle $\zeta$. This allows for the adjustment of the kinematic model to different flapping frequencies as well as a better approximation to the real wing pitch angle.

Fig. 7 a presents two possible model chord distributions indicated by the Wing frame axes $\boldsymbol{x}_{\boldsymbol{w}_{\boldsymbol{1}}}$ (dashed) and $\boldsymbol{x}_{\boldsymbol{w}_{\mathbf{2}}}$ (dottted). $\boldsymbol{x}_{\boldsymbol{w}_{\boldsymbol{1}}}$ was obtained by connecting the leading edge of the real foil distribution to the trailing edge; $\boldsymbol{x}_{\boldsymbol{w}_{\mathbf{2}}}$ was obtained in the same way, using a point placed at $50 \%$ of the wing foil.

The selection of $50 \%$ of the chord is justified by the movement of the wing during flap. It represents the evolution of the wing relative to the air flow around it, which can be observed 


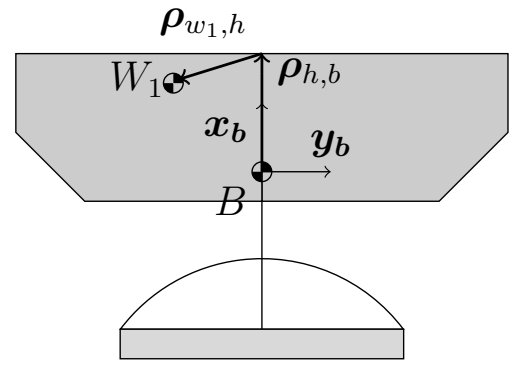

(a) Top view: $\boldsymbol{\rho}_{w_{1}, h}$ and $\boldsymbol{\rho}_{b, h}$ representation, from the CG of the body to the CG of the wing, with closed wings.

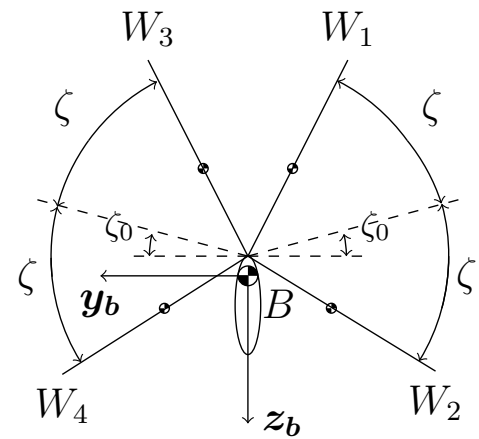

(b) Front view: wings open at angle $\zeta$; dashed line represents the position of the closed wings at $\zeta_{0}$.

Figure 6. Simplified Model with CG, Body (B) and Wing (W1 to W4) reference frames.

in Fig. 7 that represents the beginning of the out-stroke. The leading edge foil separates from the upper wing (peel motion [23]) while the after part of the foil closer to trailing edge lags considerably behind it until a $t^{*}=0.3$. Furthermore, a selection of $50 \%$ of the chord conserves the kinematic information, as the dashed line of $\boldsymbol{x}_{\boldsymbol{w}_{\mathbf{2}}}$ will better represent the position of the CG of the wing, presented in Fig. 6, thus more accurately modeling the kinematic forces that will act on the ornithopter.

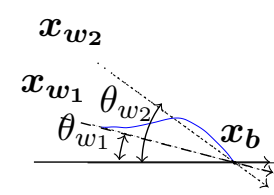

(a) $t^{*}=0.2$

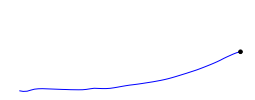

(d) $t^{*}=0.71$

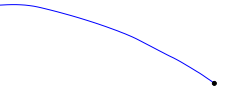

(b) $t^{*}=0.3$

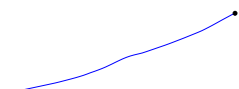

(e) $t^{*}=0.90$ (c) $t^{*}=0.48$

(f) $t^{*}=1$

Figure 7. Chordwise evolution of the airfoil during one flap cycle at $70 \%$ of the span.

Different functions were used to fit the real wing pitch angle as a function of other states. From comparing the wing pitch angle evolution with flapping in air and in vacuum it was observed that the wing deformation is more influenced by the flapping states than by other states, e.g., the free stream velocity. This way, a function (Eq. 2) was fitted to the geometric data used in Fig. 7, resulting in a model estimated wing pitch angle $\hat{\theta}_{w}$. This approach also allows for the pitch model to include only kinematic related states, thus separating kinematics from aerodynamics. 


$$
\hat{\theta}_{w}=C_{\theta_{w_{0}}}+C_{\theta_{w_{\zeta}}} \zeta+C_{\theta_{w_{\dot{\zeta}}}} \dot{\zeta}
$$

The real wing surface evolution was used to estimate the wing model pitch angle under the following assumptions:

1. the wing pitch angle data for one flap cycle is representative of all flap cycles in flight test data;

2. the wing pitch angle data measured at $70 \%$ of the span is applied on the complete span of the wing;

3. deformation of the wing in chord-wise direction is neglected;

4. all four wings have the same evolution.

The coefficients of Eq. 2 were estimated using least squares for both $\theta_{w}$ assumptions and are shown in Table 2 .

Table 2. Estimated Coefficients for Eq. 2 using two chord assumptions.

\begin{tabular}{ccc}
\hline Coefficient & $\theta_{w_{100 \%}}$ & $\theta_{w_{50 \%}}$ \\
\hline$C_{\theta_{w_{0}}}$ & 0.4476 & 0.5016 \\
$C_{\theta_{w_{\zeta}}}$ & -1.2946 & -1.5849 \\
$C_{\theta_{w_{\zeta}}}$ & -0.0160 & -0.0216 \\
\hline
\end{tabular}

The measured wing pitch angle $\left(\theta_{w}\right)$ and the estimated pitch angle $\left(\hat{\theta}_{w}\right)$ are presented in Fig. 8 for one flap cycle. The lines in full represent the measured wing pitch angle $\left(\theta_{w}\right)$ for the two chord lines $\boldsymbol{x}_{\boldsymbol{w}_{\mathbf{1}}}$ and $\boldsymbol{x}_{\boldsymbol{w}_{\mathbf{2}}}$ shown before in Fiq. 7a and the dashed lines represent the estimated wing pitch angle evolution, computed using Eq. 2.

Unlike the $\boldsymbol{x}_{\boldsymbol{w}_{\mathbf{2}}}$, the chord line $\boldsymbol{x}_{\boldsymbol{w}_{\mathbf{1}}}$ connects the leading edge to the trailing edge, thus not conserving the camber information of the wing, which is responsible for the differences in the recorded pitch angle evolution presented in Fig. 8 ,

The inflections on both evolutions of the measured pitch angle are subject to noise from the visual data acquisition, which is more pronounced in $\boldsymbol{x}_{\boldsymbol{w}_{\mathbf{2}}}$, e.g. between $t^{*} \in[0.25,0.35]$ and $t^{*} \in[0.8,0.9]$. Nevertheless, despite not perfectly following the collected data, the estimated wing pitch angle $\left(\hat{\theta}_{w}\right)$ avoids the noisy pitch oscillations and presents an evolution over one flap cycle that avoids kinematic force issues in the formulations that would come from the noisy data.

Body Frame to Wing Frames The transformation from the Body frame to each of the four Wing frames consists of a first rotation around the $\boldsymbol{x}_{\boldsymbol{b}}$ axis followed by a second rotation around $\boldsymbol{y}_{\boldsymbol{w}}$, defined as: 


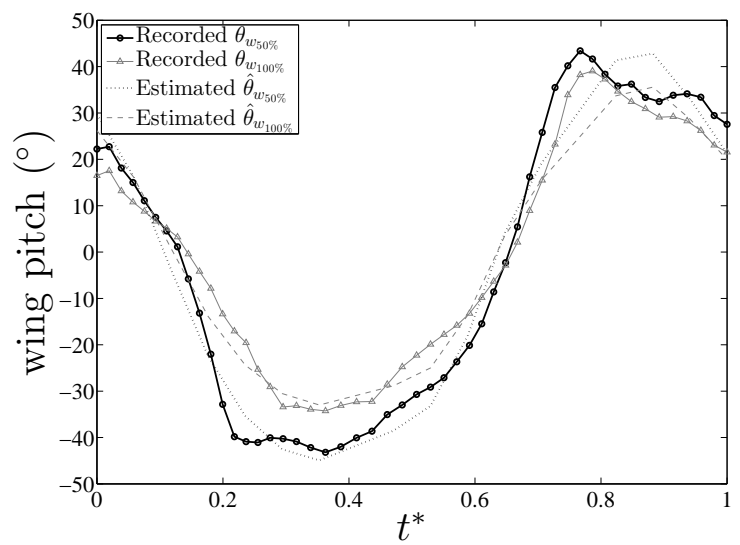

Figure 8. Recorded $\left(\theta_{w}\right)$ [22] and estimated $\left(\hat{\theta}_{w}\right)$ wing pitch angles for two chords evolutions.

$$
\begin{aligned}
& \mathcal{R}_{w 1, b}= {\left[\begin{array}{ccc}
\cos \left(\theta_{w}\right) & \sin \left(\theta_{w}\right) \sin \left(\zeta_{0}+\zeta\right) & -\sin \left(\theta_{w}\right) \cos \left(\zeta_{0}+\zeta\right) \\
0 & \cos \left(\zeta_{0}+\zeta\right) & \sin \left(\zeta_{0}+\zeta\right) \\
\sin \left(\theta_{w}\right) & -\cos \left(\theta_{w}\right) \sin \left(\zeta_{0}+\zeta\right) & \cos \left(\theta_{w}\right) \cos \left(\zeta_{0}+\zeta\right)
\end{array}\right] } \\
& \mathcal{R}_{w 2, b}=\left[\begin{array}{ccc}
\cos \left(\theta_{w}\right) & \sin \left(\theta_{w}\right) \sin \left(\zeta_{0}-\zeta\right) & -\sin \left(\theta_{w}\right) \cos \left(\zeta_{0}-\zeta\right) \\
0 & \cos \left(\zeta_{0}-\zeta\right) & \sin \left(\zeta_{0}-\zeta\right) \\
\sin \left(\theta_{w}\right) & -\cos \left(\theta_{w}\right) \sin \left(\zeta_{0}-\zeta\right) & \cos \left(\theta_{w}\right) \cos \left(\zeta_{0}-\zeta\right)
\end{array}\right] \\
& \mathcal{R}_{w 3, b}=\left[\begin{array}{ccc}
\cos \left(\theta_{w}\right) & \sin \left(\theta_{w}\right) \sin \left(-\zeta_{0}-\zeta\right) & -\sin \left(\theta_{w}\right) \cos \left(-\zeta_{0}-\zeta\right) \\
0 & \cos \left(-\zeta_{0}-\zeta\right) & \sin \left(-\zeta_{0}-\zeta\right) \\
\sin \left(\theta_{w}\right) & -\cos \left(\theta_{w}\right) \sin \left(-\zeta_{0}-\zeta\right) & \cos \left(\theta_{w}\right) \cos \left(-\zeta_{0}-\zeta\right)
\end{array}\right] \\
& \mathcal{R}_{w 4, b}=\left[\begin{array}{ccc}
\cos \left(\theta_{w}\right) & \sin \left(\theta_{w}\right) \sin \left(-\zeta_{0}+\zeta\right) & -\sin \left(\theta_{w}\right) \cos \left(-\zeta_{0}+\zeta\right) \\
0 & \cos \left(-\zeta_{0}+\zeta\right) & \sin \left(-\zeta_{0}+\zeta\right) \\
\sin \left(\theta_{w}\right) & -\cos \left(\theta_{w}\right) \sin \left(-\zeta_{0}+\zeta\right) & \cos \left(\theta_{w}\right) \cos \left(-\zeta_{0}+\zeta\right)
\end{array}\right]
\end{aligned}
$$

The position of the $\mathrm{CG}$ of each wing is first defined in its own Wing frame ( $W_{1}$ to $\left.W_{4}\right)$ as $\boldsymbol{\rho}_{w, h}$ (Fig. 6) and then transformed to the Body frame using the transpose of the transformation matrices found in Eq. 3, resulting in:

$$
\boldsymbol{\rho}_{b_{w i, h}}=\mathcal{R}_{b, w_{i}} \boldsymbol{\rho}_{w_{i}, h} \quad \text { with } \quad i=1,2,3,4
$$

INERTIAL FRAME TO BODY FRAME The transformation from the Inertial frame to the Body frame is done using quaternions and the rotation matrix takes the form [24]: 


$$
\mathcal{R}_{b, I}=\left[\begin{array}{ccc}
e_{1}^{2}+e_{0}^{2}-e_{2}^{2}-e_{3}^{2} & 2\left(e_{1} e_{2}+e_{3} e_{0}\right) & 2\left(e_{1} e_{3}-e_{2} e_{0}\right) \\
2\left(e_{1} e_{2}-e_{3} e_{0}\right) & -e_{2}^{2}-e_{0}^{2}+e_{1}^{2}+e_{3}^{2} & 2\left(e_{2} e_{3}+e_{1} e_{0}\right) \\
2\left(e_{1} e_{3}+e_{2} e_{0}\right) & 2\left(e_{2} e_{3}-e_{1} e_{0}\right) & -e_{3}^{2}-e_{0}^{2}+e_{1}^{2}+e_{2}^{2}
\end{array}\right]
$$

The angular velocities in the Body frame can now be determined using:

$$
\left[\begin{array}{l}
0 \\
p \\
q \\
r
\end{array}\right]=2\left[\begin{array}{cccc}
e_{0} & e_{1} & e_{2} & e_{3} \\
-e_{1} & e_{0} & e_{3} & -e_{2} \\
-e_{2} & -e_{3} & e_{0} & e_{1} \\
-e_{3} & e_{2} & -e_{1} & 0
\end{array}\right]\left[\begin{array}{c}
\dot{e_{0}} \\
\dot{e_{1}} \\
\dot{e_{2}} \\
\dot{e_{3}}
\end{array}\right]
$$

Mass, CG Position and Inertia Moments The full FWMAV and wing CG positions in the Body frame were $\left(x_{b}, y_{b}, z_{b}\right)=(-77,0,5.5) \mathrm{mm}$ and $\left(x_{b}, y_{b}, z_{b}\right)=(-16.5, \pm 67,0) \mathrm{mm}$, respectively measured from the hinge point of the wings, located at the nose. This position can be seen in Fig. 6. The mass and moment of inertia along the main axes of all rigid bodies is presented in Table 3 .

Table 3. Mass and Moments of Inertia of Each Modeled Body

\begin{tabular}{lccc}
\hline & Complete DelFly & Body & Wing $(\mathrm{x} 4)$ \\
\hline Mass $(\mathrm{g})$ & 17.4 & 16.2 & 0.298 \\
$I_{x x}\left[\mathrm{Kgm}^{2}\right]$ & $1.34 E^{-05}$ & $5.94 E^{-06}$ & $4.44 E^{-07}$ \\
$I_{y y}\left[\mathrm{Kgm}^{2}\right]$ & $6.58 E^{-05}$ & $6.29 E^{-05}$ & $1.74 E^{-07}$ \\
$I_{z z}\left[\mathrm{Kgm}^{2}\right]$ & $6.95 E^{-05}$ & $6.27 E^{-05}$ & $6.18 E^{-07}$ \\
\hline
\end{tabular}

Only the diagonal terms of the wing inertia tensor were used and transformed to the Body frame using the inverse of Eq. 3:

$$
I_{w}^{b}=\mathcal{R}_{b, w} I_{w} \mathcal{R}_{b, w}^{T}
$$

\section{Mathematical Formulation}

Generalized coordinates and Quasi-Velocities The main body has six d.o.f. in Inertial space which then translate into seven generalized coordinates $\left(q_{j}\right)$ [25] given the quaternion-based rotation of Eq. 5, the four wings $\left(W_{1}\right.$ to $\left.W_{4}\right)$ have two extra d.o.f., $\zeta$ and $\theta_{w}$. However, since $\theta_{w}$ is correlated $\zeta$ by Eq. 2 this results in only one added generalized coordinate. Hence, there are eight generalized coordinates to be considered in the kinematic equations: the inertial position of the main body $(x, y, z)$, the quaternion terms $\left(e_{0}, e_{1}, e_{2}, e_{3}\right)$ and the flap angle $\zeta$. 


$$
\boldsymbol{q}_{j}=\left[x, y, z, e_{0}, e_{1}, e_{2}, e_{3}, \zeta\right]
$$

There are also eight generalized velocities, which correspond to the time derivatives of $\boldsymbol{q}_{j}$. Nevertheless, there are only seven quasi-velocities thanks to one non-holonomic [25] constraint in the generalized coordinates, caused by the quaternion definition. Consequently, the quasi-velocities are formed by the linear velocities $(u, v, w)$, the angular velocities $(p, q, r)$ and the time derivative of the flapping angle $(\dot{\zeta})$, as follows:

$$
\boldsymbol{u}_{j}=[u, v, w, p, q, r, \dot{\zeta}]
$$

Velocities The translational velocities for each rigid body written in the Body frame are:

$$
\begin{aligned}
& \boldsymbol{v}_{1}=u \boldsymbol{x}_{b}+v \boldsymbol{y}_{b}+w \boldsymbol{z}_{b} \\
& \boldsymbol{v}_{i}=\boldsymbol{v}_{1}+\boldsymbol{\omega}_{1} \times \boldsymbol{\rho}_{h, b}+\boldsymbol{\omega}_{i} \times \boldsymbol{\rho}_{b_{w_{i-1}, h}} \text { with } i=2,3,4,5
\end{aligned}
$$

and the angular velocities are defined as:

$$
\begin{aligned}
\boldsymbol{\omega}_{1}= & p \boldsymbol{x}_{b}+q \boldsymbol{y}_{b}+r \boldsymbol{z}_{b} \\
\boldsymbol{\omega}_{2}= & (p+\dot{\zeta}) \boldsymbol{x}_{b}+\left(q-\dot{\theta}_{w} \cos \left(\zeta_{0}+\zeta\right)\right) \boldsymbol{y}_{b}+ \\
& \left(r+\dot{\theta}_{w} \sin \left(\zeta_{0}+\zeta\right)\right) \boldsymbol{z}_{b} \\
\boldsymbol{\omega}_{3}= & (p-\dot{\zeta}) \boldsymbol{x}_{b}+\left(q+\dot{\theta}_{w} \cos \left(\zeta_{0}-\zeta\right)\right) \boldsymbol{y}_{b}+ \\
& \left(r-\dot{\theta}_{w} \sin \left(\zeta_{0}-\zeta\right)\right) \boldsymbol{z}_{b} \\
\boldsymbol{\omega}_{4}= & (p-\dot{\zeta}) \boldsymbol{x}_{b}+\left(q-\dot{\theta}_{w} \cos \left(-\zeta_{0}-\zeta\right)\right) \boldsymbol{y}_{b}+ \\
& \left(r+\dot{\theta}_{w} \sin \left(-\zeta_{0}-\zeta\right)\right) \boldsymbol{z}_{b} \\
\boldsymbol{\omega}_{5}= & (p+\dot{\zeta}) \boldsymbol{x}_{b}+\left(q+\dot{\theta}_{w} \cos \left(-\zeta_{0}+\zeta\right)\right) \boldsymbol{y}_{b}+ \\
& \left(r-\dot{\theta}_{w} \sin \left(-\zeta_{0}+\zeta\right)\right) \boldsymbol{z}_{b}
\end{aligned}
$$

ACCELERAtions Given the previous definitions, the translational and linear accelerations of the main body terms become:

$$
\begin{gathered}
\dot{\boldsymbol{v}}_{1}=\frac{\partial \boldsymbol{v}_{1}}{\partial t}+\boldsymbol{\omega}_{1} \times \boldsymbol{v}_{1} \\
\dot{\boldsymbol{\omega}}_{1}=\frac{\partial \boldsymbol{\omega}_{1}}{\partial t}=\dot{p} \boldsymbol{x}_{b}+\dot{q} \boldsymbol{y}_{b}+\dot{r} \boldsymbol{z}_{b}
\end{gathered}
$$

where $\frac{\partial v_{1}}{\partial t}$ is the change in speed observed from the rotating Body frame defined in Eq. 14, 
Final Manuscript available on JOURNAL OF GUIDANCE, CONTROL, AND DYNAMICS

DOI 10.2514/1.G000923

http://arc.aiaa.org/doi/abs/10.2514/1.G000923

The second term is the acceleration caused by the rotation of the reference frame.

$$
\frac{\partial \boldsymbol{v}_{1}}{\partial t}=\dot{u} \boldsymbol{x}_{b}+\dot{v} \boldsymbol{y}_{b}+\dot{w} \boldsymbol{z}_{b}
$$

The translational and angular accelerations of wing rigid bodies are defined, respectively, by:

$$
\begin{gathered}
\dot{\boldsymbol{v}}_{i}=\frac{\partial \boldsymbol{v}_{i}}{\partial t}+\boldsymbol{\omega}_{1} \times \boldsymbol{v}_{i} \quad \text { with } \quad i=2,3,4,5 \\
\dot{\boldsymbol{\omega}}_{i}=\frac{\partial \boldsymbol{\omega}_{i}}{\partial t}+\boldsymbol{\omega}_{1} \times \boldsymbol{\omega}_{i} \quad \text { with } \quad i=2,3,4,5
\end{gathered}
$$

GEnERALIZED Forces Let $Q_{j}$ be the generalized forces related to each quasi-velocity $u_{j}$, which are defined using the virtual work principle:

$$
Q_{j}=\sum_{i=1}^{n}\left(\boldsymbol{F}_{\boldsymbol{i}} \boldsymbol{\gamma}_{\boldsymbol{i j}}+\boldsymbol{M}_{\boldsymbol{i}} \boldsymbol{\beta}_{\boldsymbol{i} \boldsymbol{j}}\right)
$$

with $n$ the number of bodies, $j$ the index of the generalized force, and $\boldsymbol{F}_{\boldsymbol{i}}$ and $\boldsymbol{M}_{\boldsymbol{i}}$ the external acting forces and moments respectively. $\boldsymbol{\gamma}_{i j}$ and $\boldsymbol{\beta}_{i j}$ respectively represent the velocity coefficient matrix and the angular velocity coefficient and can calculated by differentiating the linear and angular velocities of each body with respect to each quasi-velocity, using:

$$
\begin{gathered}
\boldsymbol{\gamma}_{i j}=\frac{\partial \boldsymbol{v}_{i}}{\partial \boldsymbol{u}_{j}} \\
\boldsymbol{\beta}_{i j}=\frac{\partial \boldsymbol{\omega}_{i}}{\partial \boldsymbol{u}_{j}}
\end{gathered}
$$

The forces $Q_{1}, Q_{2}$ and $Q_{3}$ are the resultant forces along the Body frame axes, which include gravity and aerodynamic forces (that will be calculated bellow). The weight vector is expressed in the Body frame axes by:

$$
\boldsymbol{g}_{\boldsymbol{b}}=\mathcal{R}_{b, I}\left[\begin{array}{lll}
0 & 0 & g
\end{array}\right]^{T}
$$

Hence, the first three generalized forces become:

$$
\left[\begin{array}{l}
Q_{1} \\
Q_{2} \\
Q_{3}
\end{array}\right]=\left[\begin{array}{l}
X \\
Y \\
Z
\end{array}\right]+\left(m_{1}+m_{2}+m_{3}+m_{4}+m_{5}\right) \boldsymbol{g}_{\boldsymbol{b}}
$$

The following generalized forces $\left(Q_{4}, Q_{5}\right.$ and $\left.Q_{6}\right)$ are the moments in the Body frame, 
which also include gravity $\left(\boldsymbol{M}_{\boldsymbol{g}}\right)$ and aerodynamic $(L, M, N)$ terms:

$$
\begin{gathered}
{\left[\begin{array}{l}
Q_{4} \\
Q_{5} \\
Q_{6}
\end{array}\right]=\left[\begin{array}{l}
L \\
M \\
N
\end{array}\right]+\boldsymbol{M}_{\boldsymbol{g}}\left[\begin{array}{l}
\boldsymbol{x}_{b} \\
\boldsymbol{y}_{b} \\
\boldsymbol{z}_{b}
\end{array}\right]} \\
\text { with } \boldsymbol{M}_{\boldsymbol{g}}=\sum_{i=1}^{4} \boldsymbol{\rho}_{\boldsymbol{w}_{\boldsymbol{i}}, \boldsymbol{b}} \times\left(m_{i+1} \boldsymbol{g}_{\boldsymbol{b}}\right)
\end{gathered}
$$

The last generalized force, $Q_{7}$, is the moment needed to flap the wings, that represents the motor torque needed to overcome wing kinematic loads $\left(M_{m}\right)$ :

$$
Q_{7}=M_{m}
$$

D'Alembert's Method For Rigid Bodies All the previous derivations can now be used to calculate the aerodynamic forces (Eq. 21) and moments (Eq. 22) acting on the FWMAV by replacing $Q_{j}$ in the previous formulations, using d'Alembert's dynamic principle of virtual work [25]:

$$
\sum_{i=1}^{n}\left[m_{i}\left(\dot{\boldsymbol{v}}_{\boldsymbol{i}}+\ddot{\boldsymbol{\rho}}_{c i}\right) \boldsymbol{\gamma}_{\boldsymbol{i j}}+\left(I_{i} \dot{\boldsymbol{\omega}}_{i}+\boldsymbol{\omega}_{i} \times I_{i} \boldsymbol{\omega}_{i}+m_{i} \boldsymbol{\rho}_{c i} \times \dot{v}_{i}\right) \boldsymbol{\beta}_{\boldsymbol{i j}}\right]=Q_{j}
$$

where $i$ is the body index and $j$ is the quasi-velocity or generalized force index, $m_{i}$ is the mass of each rigid body, $I_{i}$ the inertia matrices for the rigid bodies and $\boldsymbol{\rho}_{c i}$ is the vector from the reference point of a body to its center of mass. If the reference point of the rigid body is its CG $\left(\boldsymbol{\rho}_{c i}=0\right)$ the equation simplifies to:

$$
\sum_{i=1}^{N}\left[m_{i} \dot{\boldsymbol{v}}_{\boldsymbol{i}} \boldsymbol{\gamma}_{\boldsymbol{i j}}+\left(I_{i} \dot{\boldsymbol{\omega}}_{i}+\boldsymbol{\omega}_{i} \times I_{i} \boldsymbol{\omega}_{i}\right) \boldsymbol{\beta}_{\boldsymbol{i j}}\right]=Q_{j}
$$

\section{Applicability Aspects}

Such flapping wing kinematic model is able to better represent the physical FWMAV when compared to rigid body models. It accounts for mass and inertia distribution changes and separates the kinematic contribution from the aerodynamic forces and moments that are reconstructed, allowing for a more accurate aerodynamic reconstruction. It also avoids the complex differentiations of energy-based methods, e.g., using Lagrange's formulation.

Despite modeling the flexible FWMAV wings as rigid bodies, this formulation still accounts for the two d.o.f. of the wings and uses real wing chord evolution to model the movement of the rigid bodies, thus minimizing the errors between the aerodynamic recon- 
Final Manuscript available on JOURNAL OF GUIDANCE, CONTROL, AND DYNAMICS

DOI 10.2514/1.G000923

http://arc.aiaa.org/doi/abs/10.2514/1.G000923

struction of the model and the real FWMAV. Furthermore, this formulation results in a simpler computational analysis when compared to flexible multi-body formulations, which justifies its application for onboard modeling.

The errors resulting from modeling flexible structures as rigid bodies were considered to be negligible for the assessment of the validity of rigid body formulations for FWMAV aerodynamic reconstruction.

\section{Experimental Approach}

This section describes the experimental methodology and data processing techniques used for aerodynamic reconstruction of the FWMAV. Detailed information can be found in $[21,26]$.

\section{A. Experimental Techniques}

An external visual tracking system was used to capture the position of the FWMAV in free flight, in 3D space at a frequency of $200 \mathrm{~Hz}$ using eight reflective markers. The flight chamber had a volume of $(22 \times 17 \times 10) \mathrm{m}^{3}$. The markers were placed at the nose, wing trailing edge (on fuselage/body), wings (at the intersection of the stiffeners with the leading edge spar), horizontal and vertical stabilizers, as well as on the elevator and rudder, as indicated in Fig. 3 .

For the current test cases, the DelFly was specially configured with an autopilot that was able to maintain a trimmed flight condition, constant pitch angle, height and velocity throughout the flights. Inputs on the elevator, rudder and flapping motor were commanded at different frequencies inducing oscillations on the FWMAV around a trimmed flight [27].

\section{B. Flight Path Reconstruction}

The recorded data were processed using flight path reconstruction techniques [28]. A threepoint central difference method was used for calculating the velocities and accelerations, from the position and attitude data. This method reduces the noise amplification by a factor of two when compared with the two-point finite difference. To compensate for the added noise effect, which is more significant at frequencies that are several times the flap frequency, a Chebyshev type II low-pass filter was used, with $-80 \mathrm{~dB}$ attenuation and $40 \mathrm{~Hz}$ cut-off frequency. This way the more energetic contributions located at the flapping frequency and the following two harmonics were conserved, while the higher frequency noisy data was eliminated.

\footnotetext{
${ }^{a}$ All flight tests in this paper were conducted at the U.S. Air Force Research Laboratory Micro Air Vehicles Integration and Application Institute [27.
} 
Final Manuscript available on JOURNAL OF GUIDANCE, CONTROL, AND DYNAMICS

DOI 10.2514/1.G000923

http://arc.aiaa.org/doi/abs/10.2514/1.G000923

The states and input commands that were reconstructed are presented in Table 4, All states were calculated with respect to the CG of the ornithopter and were used for the calculation of the aerodynamic forces and moments from the dynamic formulations that were derived in the previous section.

Table 4. States and input commands reconstructed from flight data.

\begin{tabular}{ccccccccc}
\hline Quaternions & $\begin{array}{c}\text { Euler } \\
\text { Angles }\end{array}$ & $\begin{array}{c}\text { Euler } \\
\text { Angle } \\
\text { Rates }\end{array}$ & Velocities & Accelerations & $\begin{array}{c}\text { Angular } \\
\text { Body } \\
\text { Rates }\end{array}$ & $\begin{array}{c}\text { Angular } \\
\text { Body Accel- } \\
\text { erations }\end{array}$ & Flapping & Inputs \\
\hline$e_{0}$ & $\phi$ & $\dot{\phi}$ & $u$ & $\dot{u}$ & $p$ & $\dot{p}$ & $\zeta$ & $\delta_{f}($ flap freq. $)$ \\
$e_{1}$ & $\theta$ & $\dot{\theta}$ & $v$ & $\dot{v}$ & $q$ & $\dot{q}$ & $\dot{\zeta}$ & $\delta_{e}($ elevator $)$ \\
$e_{2}$ & $\psi$ & $\dot{\psi}$ & $w$ & $\dot{w}$ & $r$ & $\dot{r}$ & $\ddot{\zeta}$ & $\delta_{r}($ rudder $)$ \\
\hline
\end{tabular}

\section{Results and Discussion}

The current section compares the aerodynamic forces and moments reconstructed from the flight data using the dynamic models derived in Section II.

First, the control surface positions of the real FWMAV, as well as the aerodynamic forces and moments reconstructed from each dynamic model are shown. This is followed by an extension of the results to different wing-to-body mass ratios. Finally, the validity regions of the models are discussed.

\section{A. Force and Moment Identification Results}

A single part of the flight tests was considered to be representative of the flight conditions, as it covers trimmed flight and abrupt elevator change $\mathrm{b}$.

Fig. 9 presents a visualization of the trajectory of the FWMAV. It shows one second of horizontal leveled flight, followed by an elevator doublet input over $\frac{2}{3}$ seconds which induced a longitudinal oscillatory movement that was dampened after three seconds. The red and magenta parts in Fig. 9 represent the elevator deflection to maximum up (negative) and down (positive), respectively, as indicated in the middle plot of Fig. 10, at 6sec. The blue marker indicates the transition from positive to negative. The ornithopter goes over $90^{\circ}$ in pitch just before losing altitude. The arrows indicate the $\boldsymbol{x}_{\boldsymbol{b}}$ (black) and $\boldsymbol{z}_{\boldsymbol{b}}$ (gray) Body axes along the maneuver and are equally spaced of $0.2 \mathrm{sec}$. The flight patch is projected on the $x_{b} y_{b}$ plane for better visualization.

\footnotetext{
${ }^{\mathrm{b}}$ The selected flight test can be seen in the video file that accompanies the article.
} 
The loss of altitude during the maneuver is caused by the transition from forward to hovering flight, causing the lift to be smaller due to the lack of relative wind on the wings and tail. This effect is then aggravated by the elevator deflection down, which initially causes the FWMAV to lose more altitude, and then contributes to an increase in forward velocity $u$, that allows the FWMAV to regain altitude and return to leveled flight while keeping all inputs at commands trimmed position.

The influence of the displacement of the wings during flapping (top plot of Fig. 10) is visible on the sub-flap oscillations of the elevator $\left(\delta_{e}\right)$ and rudder $\left(\delta_{r}\right)$ positions, depicted in the middle and bottom plots of Fig. 10.

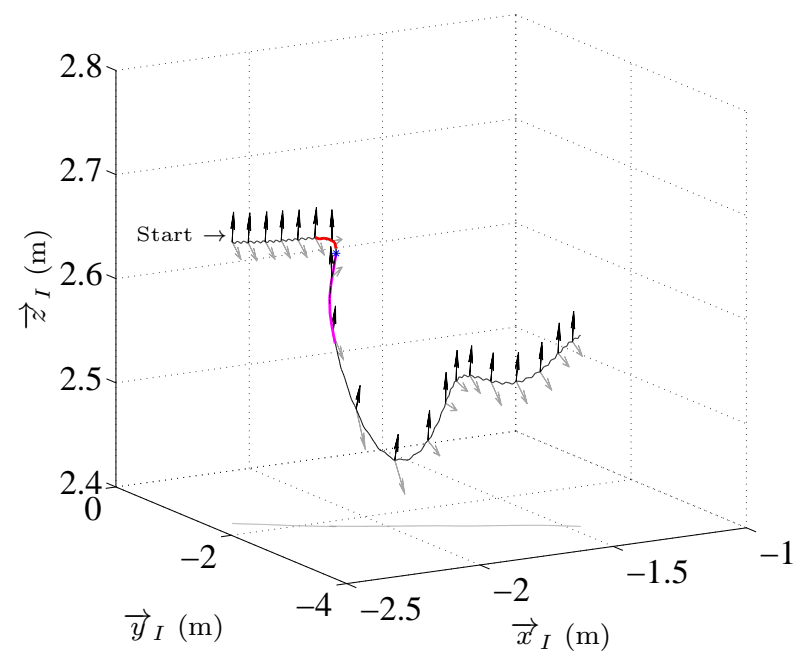

Figure 9. CG position of the FWMAV in the Inertial reference frame during the flight test.

The states in Table 4 that were reconstructed from the position and attitude of the ornithopter can be found in [21].

The evolution of the aerodynamic forces and moments, presented in Fig. 11, shows that both kinematic derivations lead to very similar aerodynamic forces and moments, even during highly nonlinear maneuvers, like the one shown.

Among the forces, the $Z$ force shows the biggest differences between both methods, while $X$ reveals an almost perfect match. The differences in $Z$ are caused by the deceleration of the wings at the end of the out-stroke, where the flap angle is maximum, since the wing stroke plane is almost parallel to the $\boldsymbol{z}_{\boldsymbol{b}}$ axis. This results in added pitch inducing forces along $\boldsymbol{z}_{\boldsymbol{b}}$ which, in turn, induce variations in the pitch moment $M$ between both methods, as observed in Fig. 11b.

The $L$ moment reveals very similar results between the methods due to the symmetry of the model along the $x_{b} z_{b}$ plane. Alike the $M$ moment, the $N$ moment calculated for the flapping kinematics has lower peaks due to separation between the kinematic and the 

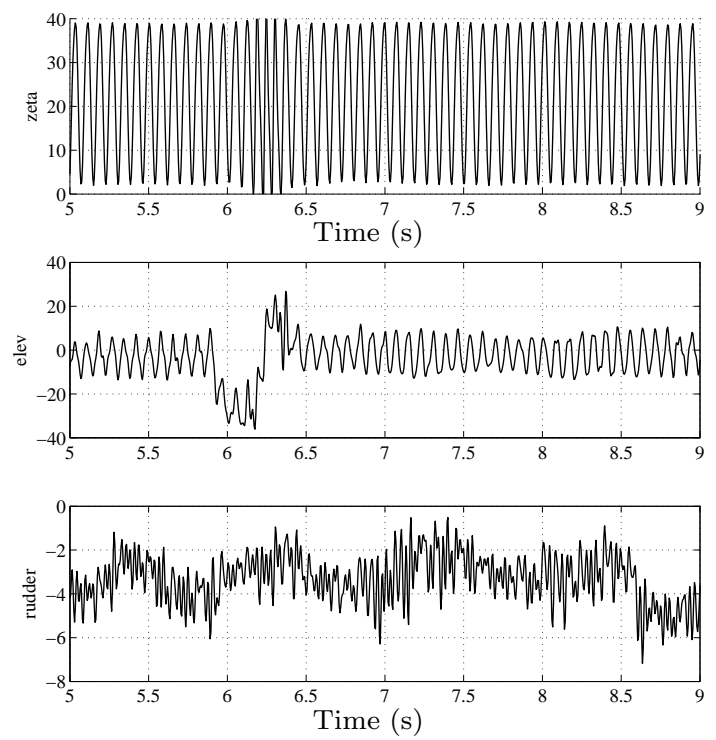

Figure 10. Inputs: flap angle $\zeta$; elevator angle deflection $\delta_{e}$; rudder angle deflection $\delta_{r}$.

aerodynamic effects of the wings.

The previous results point to the applicability of rigid body models for the aerodynamic force identification of FWMAVs that have symmetric positioning of wings and a single wingto-body mass ratio under $1.8 \%$, which is the ratio of the ornithopter that was used.

The following section explores the applicability of the kinematic models, testing the identification procedure with different wing-to-body mass ratios and wing configurations.

\section{B. Extension of Results to Different Wing-to-body Mass Ratios}

\section{Methodology Considerations}

The previous results are extended here to assess the effects of relative wing-to-body mass ratios in the aerodynamic force and moment reconstruction using the same flight data. In particular, other four-winged and two-winged ornithopter kinematics were simulated for different single wing-to-body mass ratios, while keeping the total mass of the FWMAV constant.

Body masses were changed by assuming a uniform distribution of variable density in the bodies. In effect, the inertia tensor was recalculated for each mass ratio and included in the kinematic formulations. It was assumed that the simulated FWMAVs would flap with the same frequency as the DelFly $(\sim 12 \mathrm{~Hz})$.

Root Mean Square of the Error (RMSE) and the Coefficient of Determination $\left(R^{2}\right)$ were used for the quantitative assessment of the applicability of rigid body formulations in both 

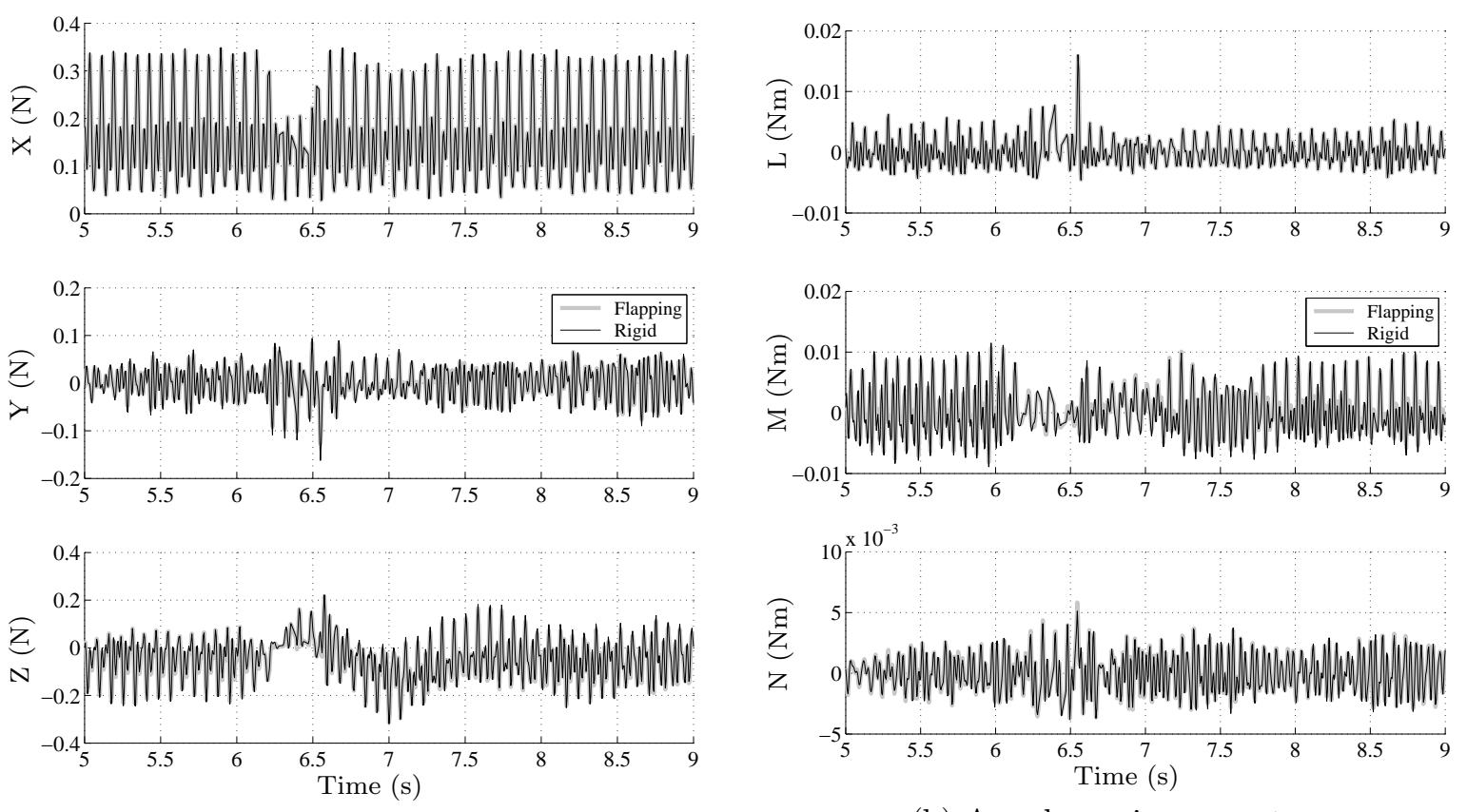

(a) Aerodynamic forces.

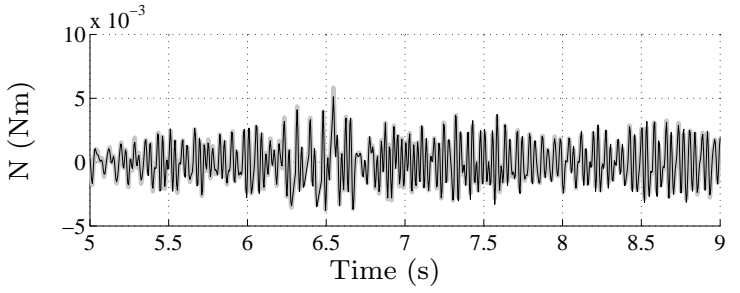

(b) Aerodynamic moments.

Figure 11. Aerodynamic forces and moments calculated using both kinematic models and physical properties of the real ornithopter.

cases. The RMSE (Eq. 27) is an indication of the absolute difference between the two signals. A value of zero indicates identical signals. The higher the value, the larger the difference between the signals.

$$
R M S E_{F_{f l a p p i n g}, F_{\text {single }}}=\sqrt{\frac{\sum\left(F_{\text {flapping }}-F_{\text {single }}\right)^{2}}{N_{i}}}
$$

It is worth noting that the aerodynamic forces and moments that were obtained with this method consist of the ones that would have acted on a FWMAV with the indicated mass and inertia properties, had it flown this trajectory. The results will be conservative since higher wing-to-body mass ratios would also induce larger body oscillations during flapping, that are not captured by the flight data. Nevertheless, this method consists of a sensitivity study and is used to predict the effect of kinematic models in aerodynamic reconstruction, thus providing a safety threshold for the application of rigid body kinematics. The fact that it uses real flight data allows for more reliable conclusions than using pure simulated trajectories based on aerodynamic models that are subjective to assumptions and approximations.

The Coefficient of Determination (Eq. 28) is the average of the squared error between two signals divided by the variance of the original signal. A value of one means a perfect fit and a value closer to zero means a bigger residual between the signals. 
Final Manuscript available on JOURNAL OF GUIDANCE, CONTROL, AND DYNAMICS

DOI 10.2514/1.G000923

http://arc.aiaa.org/doi/abs/10.2514/1.G000923

$$
R_{F_{\text {flapping }}, F_{\text {single }}}^{2}=1-\frac{\sum\left(F_{\text {flapping }}-F_{\text {single }}\right)^{2}}{\operatorname{var}\left(F_{\text {flapping }}\right) N_{i}}
$$

\section{Four-winged FWMAV}

The mass of the wings for a four-winged FWMAV is increased to obtain higher wing-to-body mass ratios and the flapping wing model is again compared with the single body model. In particular, the RMSE and the $R^{2}$ are calculated for the longitudinal $Z$ force to assess the difference between the methods, as it is the force that presents larger differences.

Table 5 shows this analysis for six different single wing-to-body mass ratios, starting from $1.8 \%$ (mass ratio of the DelFly) to $15 \%$. The differences grow with a higher wing-to-body mass ratio, observed by the decreasing $R^{2}$ and increasing RMSE - from a mass ratio of $8 \%$ the $R^{2}$ goes below 0.7 . This means that the estimated forces and moments from the flapping wing model are very different from the forces and moments estimated from a single rigid body model. Hence, using single rigid body dynamics for higher mass ratios may result in inadequate system identification results.

The forces and moments of both methods that would act on a four-winged vehicle with mass ratio of $8 \%$ are shown in Fig. 12 .

Table 5. Comparison between Single Body and Flapping Models for four-winged FWMAV.

\begin{tabular}{lccccc}
\hline Test & $\begin{array}{c}\text { Body } \\
\text { Mass (g) }\end{array}$ & $\begin{array}{c}\text { Single } \\
\text { Wing } \\
\text { Mass (g) }\end{array}$ & $\begin{array}{c}\text { Mass } \\
\text { Ratio } \\
(\%)\end{array}$ & $\begin{array}{c}\text { RMSE Z } \\
\text { Force (N) }\end{array}$ & $\begin{array}{c}\boldsymbol{R}^{\mathbf{2}} \mathbf{Z} \\
\text { Force }\end{array}$ \\
\hline 1 (real) & 16.2 & 0.298 & 1.8 & 0.0114 & 0.9806 \\
2 & 15.5 & 0.466 & 3 & 0.0183 & 0.9478 \\
3 & 14.5 & 0.725 & 5 & 0.0284 & 0.8652 \\
4 & 13.2 & 1.05 & 8 & 0.0413 & 0.6992 \\
5 & 12.4 & 1.24 & 10 & 0.0487 & 0.5770 \\
6 & 10.9 & 1.63 & 15 & 0.0639 & 0.2838 \\
\hline
\end{tabular}

For a better visual interpretation of the values presented in Table 5, the residuals in the forces and moments between both kinematic formulations for different wing to body mass ratios are presented in Fig. 13. To maximize figure readability only four ratios were included, which cover all the cases of Table 5 .

For visual interpretation, the residuals between the forces and moments calculated 
Final Manuscript available on JOURNAL OF GUIDANCE, CONTROL, AND DYNAMICS

DOI 10.2514/1.G000923

http://arc.aiaa.org/doi/abs/10.2514/1.G000923
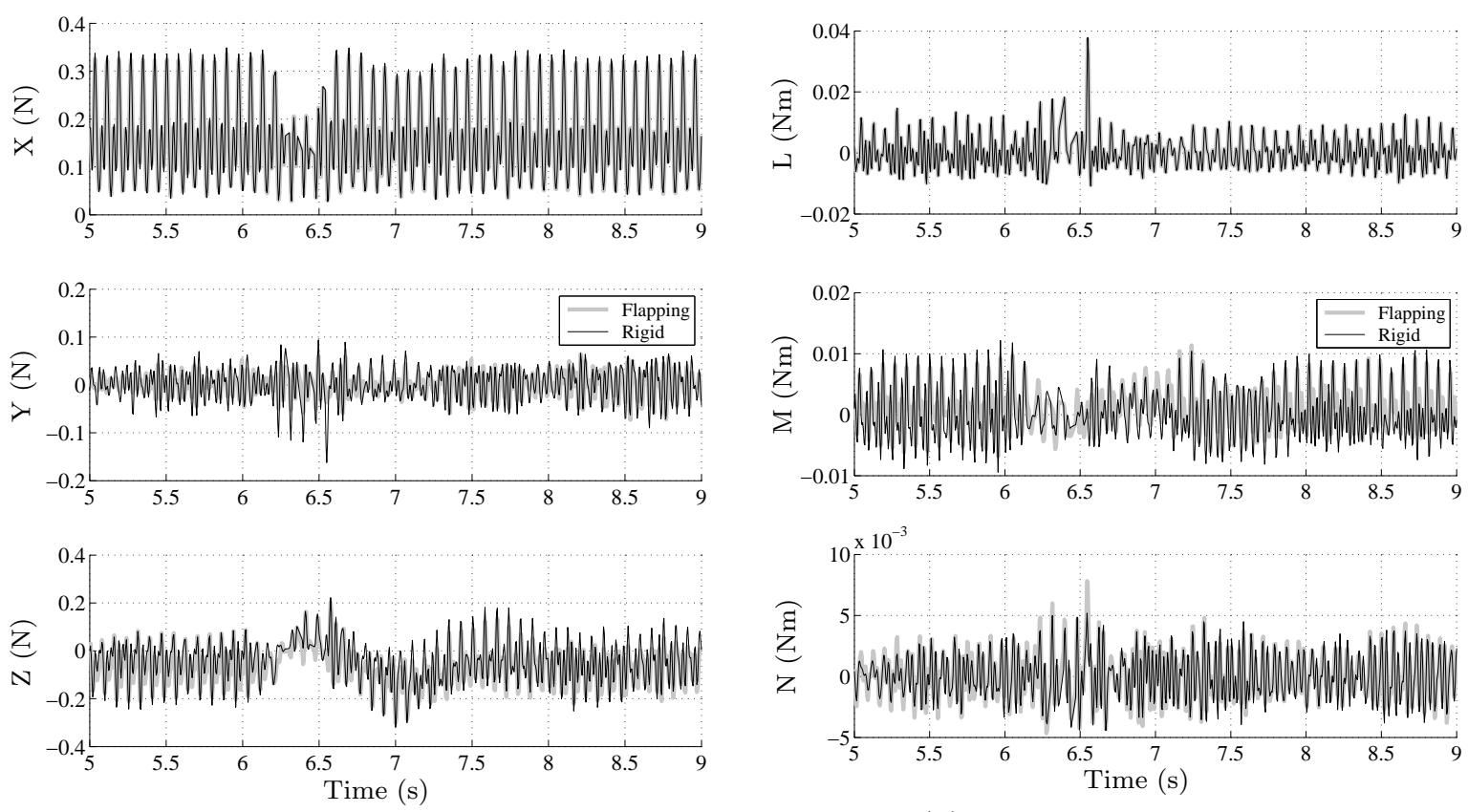

(a) Aerodynamic forces.

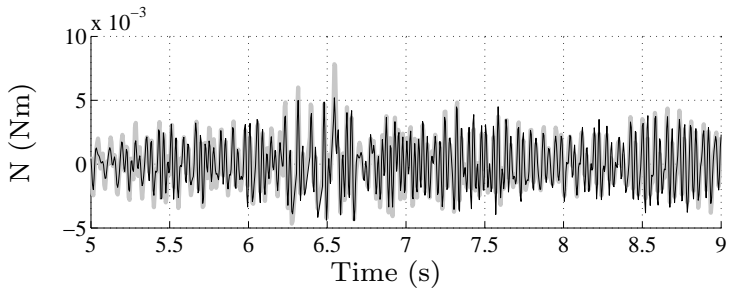

(b) Aerodynamic moments.

Figure 12. Aerodynamic forces (a) and moments (b) calculated using both kinematic models for a four-winged ornithopter with a single wing to body mass ratio of $8 \%$.
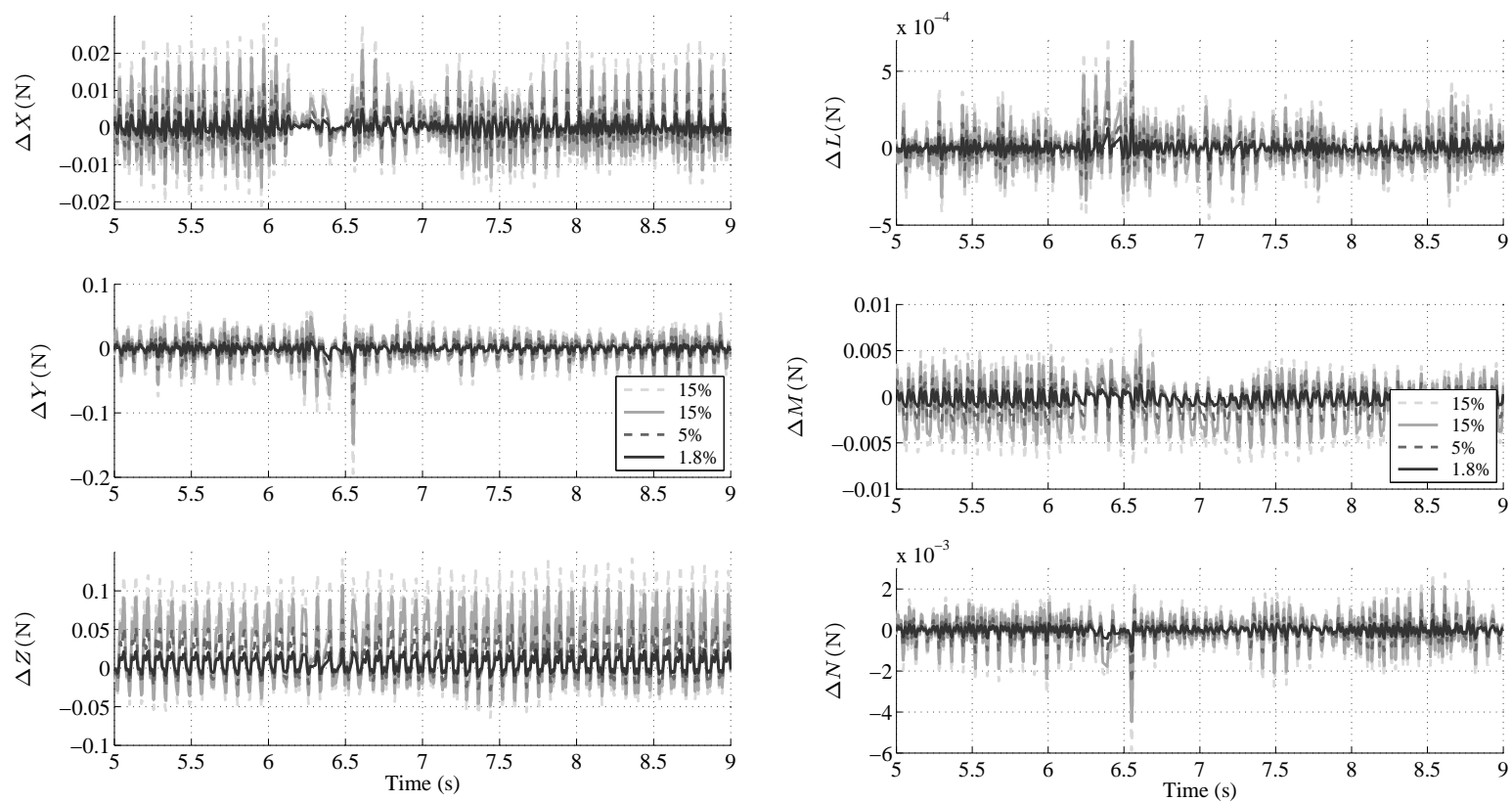

(a) Residuals of aerdoynamic forces.

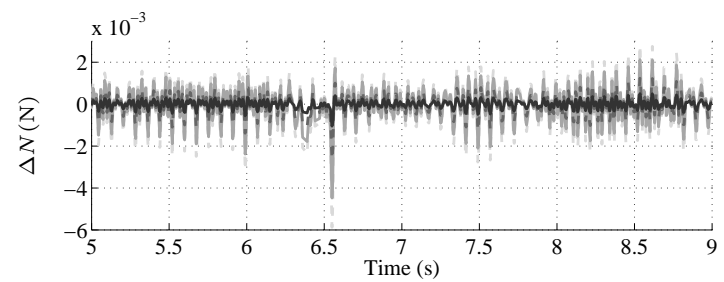

(b) Residuals of aerdoynamic moments

Figure 13. Residuals of the aerodynamic forces (a) and moments (b) between both kinematic models, for different single wing to body mass ratios, from $1.8 \%$ to $15 \%$, for a four-winged FWMAV. 
Final Manuscript available on JOURNAL OF GUIDANCE, CONTROL, AND DYNAMICS

DOI 10.2514/1.G000923

http://arc.aiaa.org/doi/abs/10.2514/1.G000923

\section{Two-winged FWMAV}

A similar analysis was done for two-winged FWMAVs, where the mass and moments of inertia of wings two and four (bottom wings) were set to zero, thus isolating the kinematic effect of the two top wings, representing a bird-like FWMAV. Given the absence of valid aerodynamic models to adequately simulate the flight trajectories, the flight data of the real ornithopter was used for assessing the effects of varying the wing-to-body mass ratio. Instead of comparing two-winged to four-winged configurations per se, this approach estimates the impact of wing-to-body mass ratio in the difference between the forces and moments obtained by both kinematic models,. Nevertheless, it conserves the impact of the kinematic model in the parametric study and allows for the understanding of the errors of using rigid body kinematics to model a FWMAV of such configuration. For the ease of comparison, the wing-to-body mass ratios used were the same as for the four-winged FWMAV.

The results, presented in Table 6, indicate that the differences in the forces and moments increase more rapidly for the same wing-to-body mass ratios - the $R^{2}$ takes values lower than 0.8 from a mass ratio above $3 \%$, highlighting the stabilizing effect of a four wing configuration similar to the real ornithopter. The forces and moments acting on a two-winged FWMAV with mass ratio $3 \%$ are shown in Figs. 14. As observed, the $Z$ force and $M$ moment present the biggest variations among the methods.

Table 6. Comparison between Single Body and Flapping Models for two-winged FWMAV.

\begin{tabular}{lccccc}
\hline Test & $\begin{array}{c}\text { Body Mass } \\
(\mathrm{g})\end{array}$ & $\begin{array}{c}\text { Single } \\
\text { Wing } \\
\text { Mass (g) }\end{array}$ & $\begin{array}{c}\text { Mass } \\
\text { Ratio (\%) }\end{array}$ & $\begin{array}{c}\text { RMSE Z } \\
\text { Force (N) }\end{array}$ & $\begin{array}{c}\boldsymbol{R}^{2} \mathbf{Z} \\
\text { Force }\end{array}$ \\
\hline 1 & 16.8 & 0.302 & 1.8 & 0.0492 & 0.8182 \\
2 & 16.4 & 0.49 & 3 & 0.0801 & 0.6687 \\
3 & 15.8 & 0.79 & 5 & 0.1287 & 0.4931 \\
4 & 15.0 & 1.19 & 8 & 0.1953 & 0.3468 \\
5 & 14.5 & 1.45 & 10 & 0.2360 & 0.2892 \\
6 & 13.4 & 2.01 & 15 & 0.3268 & 0.2062 \\
\hline
\end{tabular}

The residuals of the aerodynamic forces and moments calculated between kinematic both models of a two-winged ornithopter are presented in Fig. 15. The magnitude of the residuals shows that small changes in the wing to body mass ratios can result in considerable offset of the forces calculated using rigid body model, when compared to a flapping multi-rigid body kinematic model. 
Final Manuscript available on JOURNAL OF GUIDANCE, CONTROL, AND DYNAMICS

DOI 10.2514/1.G000923

http://arc.aiaa.org/doi/abs/10.2514/1.G000923
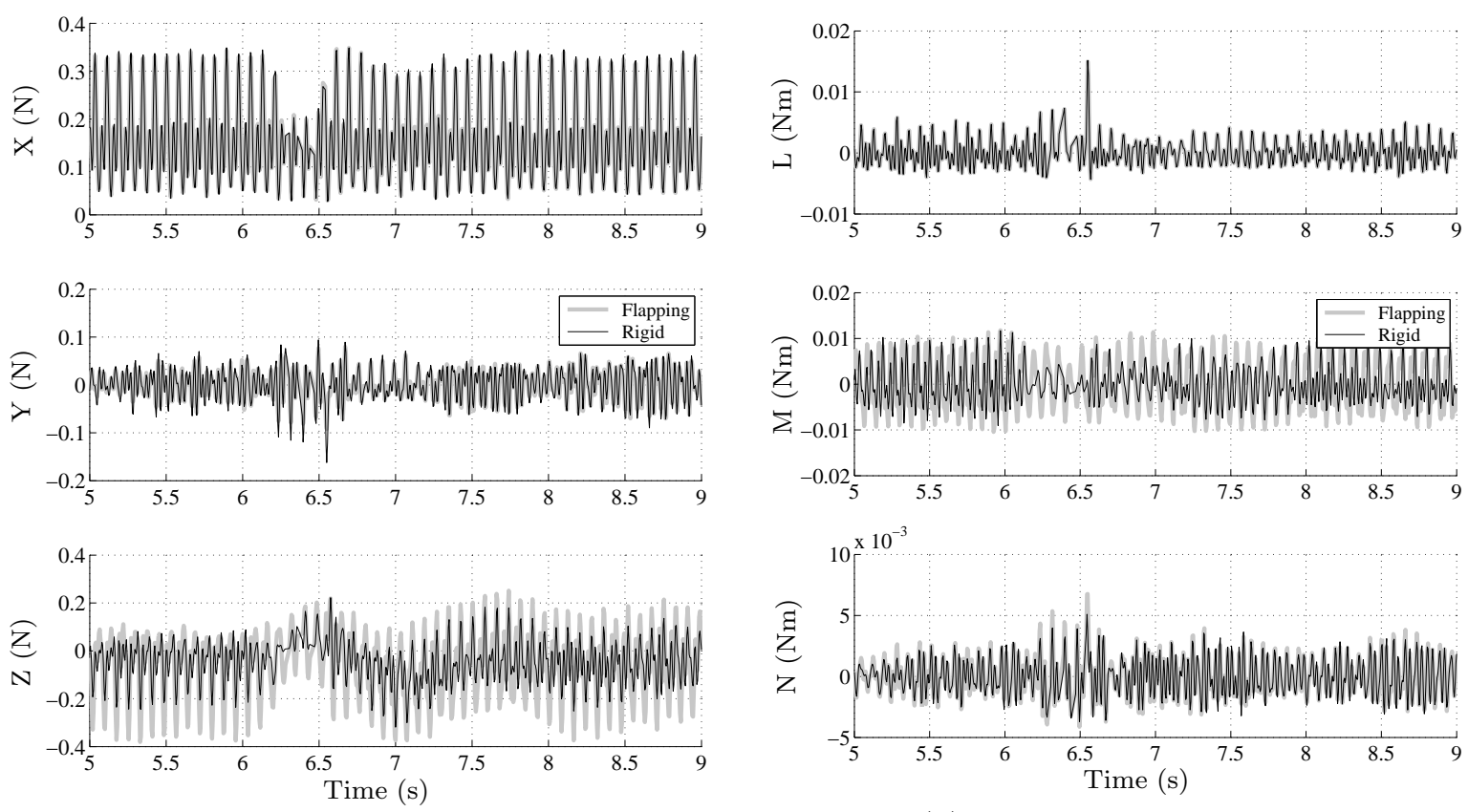

(a) Aerodynamic forces.

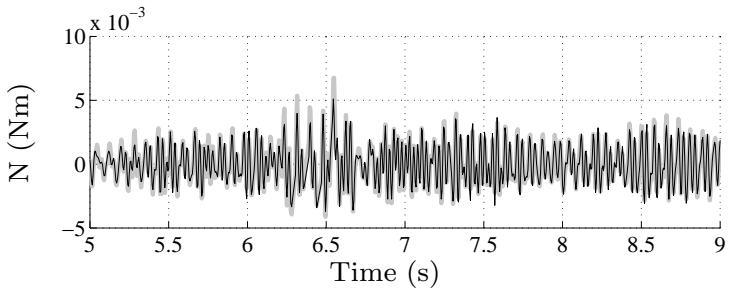

(b) Aerodynamic moments.

Figure 14. Aerodynamic forces (a) and moments (b) calculated using both kinematic models for a two-winged ornithopter with a single wing to body mass ratio of $3 \%$.
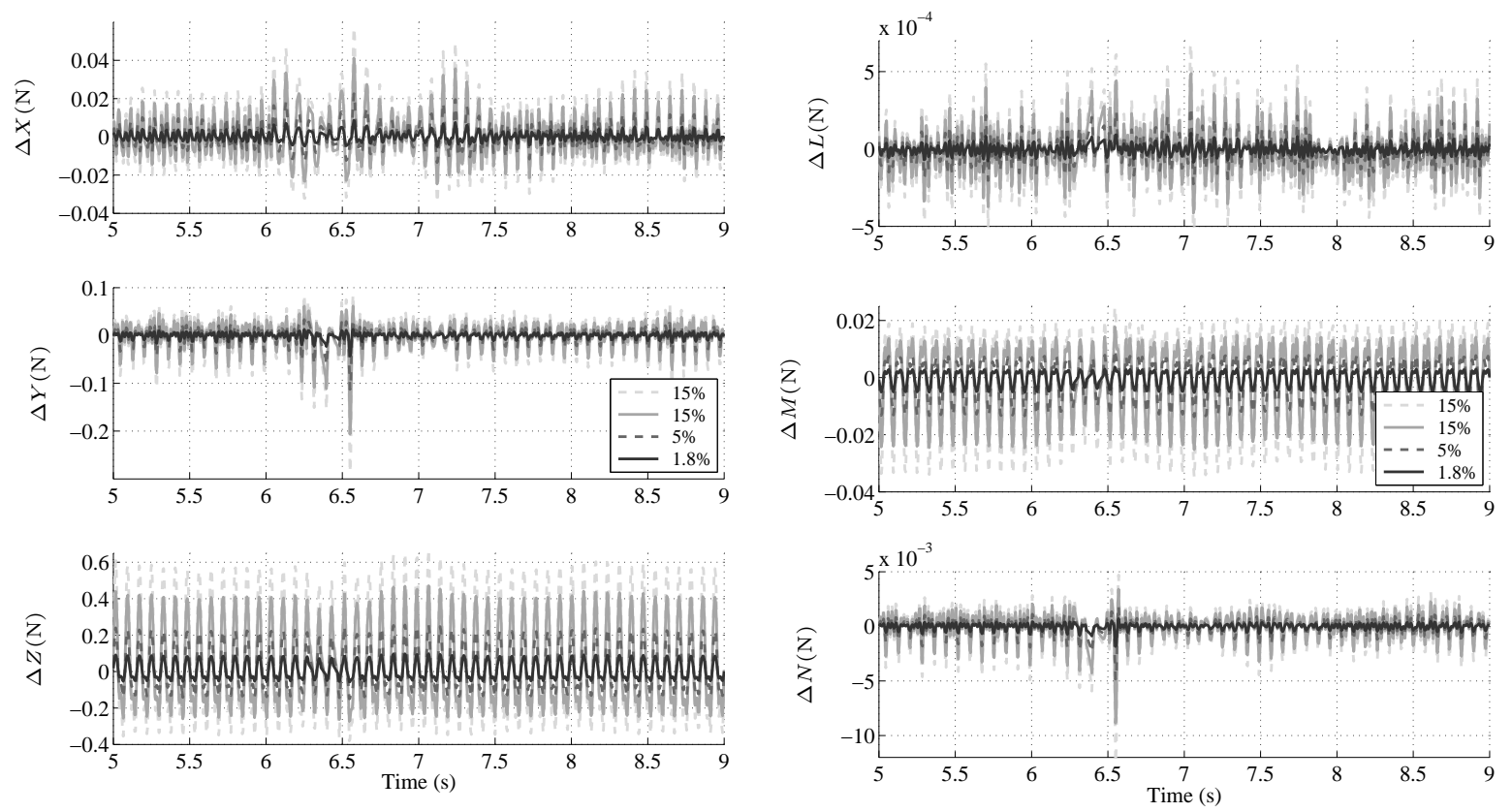

(a) Residuals of aerdoynamic forces.

(b) Residuals of aerdoynamic moments

Figure 15. Residuals of the aerodynamic forces (a) and moments (b) between both kinematic models, for different single wing to body mass ratios, from $1.8 \%$ to $15 \%$, for a two-winged FWMAV. 


\section{Discussion}

The values of $R^{2}$ presented in Tables [5] and 6] are shown in the plot of Fig. [16 for easier comparison. For the current study, an $R^{2}$ above 0.7 was considered as being acceptable for the use of rigid body kinematics for FWMAV force reconstruction. Under this criterion, single rigid body equations of motion can be used for force and moment reconstruction of free flying FWMAVs, without considerable information loss, for cases where the flap induced oscillations are small. This is the case of ornithopters with four-wings and also two-winged ones whose single wing mass to body ratios are smaller than $<8 \%$ and $<2.8 \%$, respectively (Fig. 16).

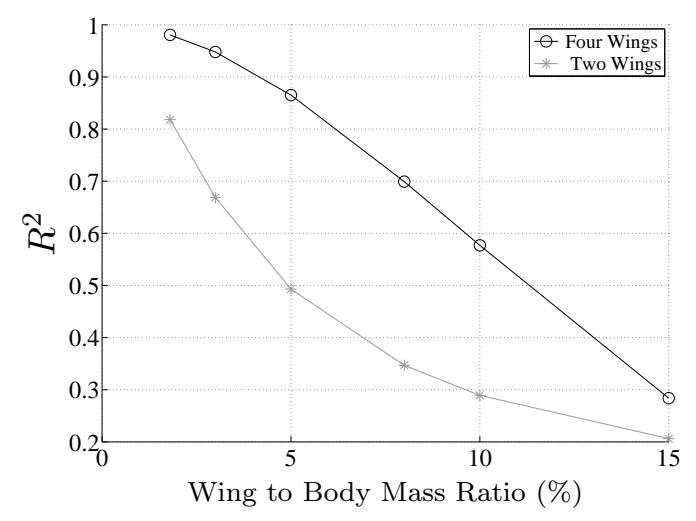

Figure 16. Coefficient of Determination of the $Z$ force with mass ration for both configurations.

These results ease the aerodynamic identification procedures since single rigid body kinematics are more easily derived than multi-body ones which subsequently shortens the development time of aerodynamic models.

Despite more complex, detailed multi-body kinematics allow for the direct assessment of control forces and moments over the individual wings and driving mechanisms, with applicability advantages for FWMAVs with active control of more than one d.o.f. of the wings, as the case of insect inspired tailless designs. In the present case, a seventh generalized force $Q_{7}$ could be calculated which describes the flapping motor torque.

Multi-body formulations also allowed for the separation between kinematic and aerodynamic forces and moments in the identification procedure, thus providing an assessment on the validity regions of rigid body formulations, allowing for more correct system identification in regions where rigid body formulations are not applicable. Furthermore, the use of quaternions has beneficial effects in computational efficiency, as well as in avoiding singularity issues of the Euler angles. 


\section{Conclusion}

This study compared the aerodynamic forces and moments computed from free flight data using two kinematic formulations devised under different assumptions: a) single rigid body equations of motion, using Newton-Euler formulation, commonly know as the general aircraft equations of motion and b) multi-rigid body flapping wing kinematics using the virtual work principle. In addition, it analyzed the extension of the first method to different Flapping Wing Micro Aerial Vehicles (FWMAVs) that had different wing-to-body mass ratios.

It was observed that more descriptive formulations that use multi-rigid body kinematics are suitable for complex FWMAVs, especially if they have more than one degree of freedom on the wings or are tailless designs, as these formulations allow for control force and moment determination, which is required for active control and dynamic simulation of such configurations. However, single rigid body approaches are simpler to devise, being useful for tailed or passively stable designs and for iterative studies of constantly changing FWMAV configurations, as these formulations still provide correct aerodynamic reconstruction for FWMAVs with low wing-to-body mass ratios without the need for costly derivations.

The results justify the use of single rigid body kinematics, here described as general aircraft equations of motion, for the reconstruction of aerodynamic forces and dynamic behavior of FWMAVs that have a single wing mass to body mass ratio under $8 \%$ for ' $\mathrm{X}$ ' wing configurations and under $2.8 \%$ for two-winged FWMAVs, equivalent to $24 \%$ and $5.6 \%$ of total ornithopter mass being at the wings for each configuration, respectively.

These results allow for the understanding of the effects of using simple rigid body kinematic models for force reconstruction of FWMAV and, consequently, ease the characterization of ornithopters with similar configurations [18, 19, 29 31], or ornithopters inspired in some birds or insect species, e.g., Pomatorhinus Ruficollis, Coturnix Coturnix [15], Schistocerca Gregaria [16], Manduca Sexta [17].

\section{Acknowledgments}

The authors thank the United States Air Force Research Laboratory Micro Air Vehicles Integration and Application Institute personnel for the all the precious help provided

during flight testing, as well as, the Fundação para a Ciência e Tecnologia for the grant $\mathrm{SFRH} / \mathrm{BD} / 80374 / 2011$.

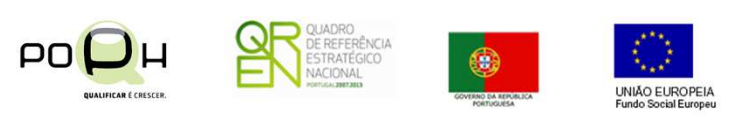


Final Manuscript available on JOURNAL OF GUIDANCE, CONTROL, AND DYNAMICS

DOI 10.2514/1.G000923

http://arc.aiaa.org/doi/abs/10.2514/1.G000923

\section{References}

${ }^{1}$ Keennon, M., Klingebiel, K., and Won, H., "Development of the Nano Hummingbird: A Tailless Flapping Wing Micro Air Vehicle," 50TH AIAA Aerospace Sciences Meeting incl. New Horizons Forum and Aerospace Exposition, No. AIAA 2012-0588, 2012, pp. 1-24. doi 10.2514/6.2012-588.

${ }^{2} \mathrm{Ma}, \mathrm{K}$., Chirarattananon, P., Fuller, S., and Wood, R., "Controlled Flight of a Biologically Inspired, Insect-Scale Robot," Science, Vol. 340, No. 6132, 2013, pp. 603-607. doi 10.1126/science.1231806.

${ }^{3}$ Hines, L. L., Arabagi, V., and Sitti, M., "Free flight simulations and pitch and roll control experiments of a sub-gram flapping-flight micro aerial vehicle," Proceedings - IEEE International Conference on Robotics and Automation, Shanghai, China, May 2011. doi 10.1109/ICRA.2011.5979816.

${ }^{4}$ Baek, S. and Fearing, R., "Flight forces and altitude regulation of 12 gram i-bird," IEEE RAS and EMBS International Conference on Biomedical Robotics and Biomechatronics (BioRob), 2010, pp. 454-460. doi:10.1109/BIOROB.2010.5626347.

${ }^{5}$ de Croon, G., de Clercq, K., Ruijsink, R., Remes, B., and de Wagter, C., "Design, aerodynamics, and vision-based control of the DelFly," International Journal of Micro Air Vehicles, Vol. 1, No. 2, 2009, pp. 71-97. doi $10.1260 / 175682909789498288$.

${ }^{6}$ Gebert, G. and Gallmeier, P., "Equations of Motion for Flapping Flight," AIAA Atmospheric Flight Mechanics Conference and Exhibit, No. 2002-4872, August 2002. doi 10.2514/6.2002-4872.

${ }^{7}$ Bolender, M. A., "Rigid Multi-Body Equations-of-Motion for Flapping Wing MAVs using Kane Equations," AIAA Guidance, Navigation, and Control Conference, No. 2009-6158, August 2009. doi: $10.2514 / 6.2009-6158$.

${ }^{8}$ Grauer, J., Ulrich, E., Jr., J. H., Pines, D., , and Humbert, J. S., "Testing and System Identification of an Ornithopter in Longitudinal Flight," Journal of Aircraft, Vol. 48, No. 2, March-April 2011, pp. 660-667. doi:10.2514/1.C031208.

${ }^{9}$ Orlowski, C. T., Girard, A. R., and Shyy, W., "Four Wing Flapping Micro Air Vehicles - Dragonfies or X-Wings?" AIAA Guidance, Navigation, and Control Conference, No. AIAA 2010-7707, Toronto, Ontario, Canada, August 2010. doi 10.2514/6.2010-7707.

${ }^{10}$ Orlowski, C. T. and Girard, A. R., "Modeling and Simulation of Nonlinear Dynamics of Flapping Wing Micro Air Vehicles," AIAA Journal, Vol. 49, No. 5, May 2011, pp. 969-981. doi 10.2514/1.J050649.

${ }^{11}$ Dietl, J. M. and Garcia, E., "Stability in Ornithopter Longitudinal Flight Dynamics," Journal of Guidance, Control and Dynamics, Vol. 31, No. 4, 2008, pp. 1157-1162. doi:10.2514/1.33561.

${ }^{12}$ Dietl, J. M., Herrmann, T., Reich, G., and Garcia, E., "Dynamic Modeling, Testing, and Stability Analysis of an Ornithoptic Blimp," Journal of Bionic Engineering, Vol. 8, No. 4, 2011, pp. 375-386. doi:10.1016/S1672-6529(11)60043-7.

${ }^{13}$ Grauer, J., Ulrich, E., Jr., J. H., Pines, D., and Humbert, J. S., "System Identification of an Ornithopter Aerodynamic Model," AIAA Atmospheric Flight Mechanics Conference, No. 2010-7632, August 2010. doi: $10.2514 / 6.2010-7632$.

${ }^{14}$ Caetano, J. V., Weehuizen, M., de Visser, C. C., de Croon, G., de Wagter, C., Remes, B., and Mulder, M., "Rigid vs. Flapping: The Effects of Kinematics Formulations in Force Determination of a Free Flying Flapping Wing Micro Air Vehicle," International Conference on Unmanned Systems, ICUAS14, 2014. doi:10.1109/ICUAS.2014.6842345.

${ }^{15}$ van den Berg, C. and Rayner, J. M., "The Moment of Inertia of Bird Wings and the Inertial Power Requirement for Flapping Flight," The Journal of Experimental Biology, Vol. 198, 1995, pp. 1655-1664. 
Final Manuscript available on JOURNAL OF GUIDANCE, CONTROL, AND DYNAMICS

DOI 10.2514/1.G000923

http://arc.aiaa.org/doi/abs/10.2514/1.G000923

${ }^{16}$ Taylor, G. K. and Thomas, A. L. R., "Dynamic flight stability in the desert locust Schistocerca gregaria," The Journal of Experimental Biology, Vol. 206, No. 16, 2003, pp. $2803-2829$. doi:doi:10.1242/jeb.00501.

${ }^{17}$ Wang, G. J. B. Z. J., "Energy-minimizing kinematics in hovering insect flight," Journal of Fluid Mechanics, Vol. 582, 2007, pp. 153-168.

18 "Wright State University Center for Excellence in Micro Air Vehicle Research," 2010.

${ }^{19}$ Rose, C. and Fearing, R. S., "Comparison of Ornithopter Wind Tunnel Force Measurements with Free Flight," 2014 IEEE International Conference on Robotics $\&$ Automation (ICRA), IEEE, May-June 2014, pp. 1816-1821. doi:10.1109/ICRA.2014.6907097.

${ }^{20}$ Stevens, B. L. and Lewis, F. L., Aircraft Control and Simulation, Jonh Wiley \& Sons, Inc., 2nd ed., 2003, p. 110.

${ }^{21}$ Caetano, J. V., de Visser, C., de Croon, G., Remes, B., de Wagter, C., Verboom, J., and Mulder, M., "Linear Aerodynamic Model Identification of a FlappingWing MAV Based on Flight Test Data," International Journal of Micro Air Vehicles, Vol. 5, No. 4, December 2013, pp. $273-286$. doi:10.1260/1756-8293.5.4.273.

${ }^{22}$ Groen, M., Bruggeman, B., Remes, B., Ruijsink, R., van Oudheusden, B., and Bijl, H., "Improving flight performance of the flapping wing MAV DelFly II," International Competition and Conference on Micro Air Vehicles, IMAV 2012, IMAV, 2010.

${ }^{23}$ de Croon, G. C. H. E., Groen, M. A., Wagter, C. D., Remes, B., Ruijsink, R., and van Oudheusden, B. W., "Design, aerodynamics and autonomy of the DelFly," Bioinspiration E Biomimetics, Vol. 7, No. 2, 2012, pp. 025003.

${ }^{24}$ Phillips, W. F., Hailey, C. E., and Gebert, G. a., "Review of Attitude Representations Used for Aircraft Kinematics," Journal of Aircraft, Vol. 38, No. 4, July 2001, pp. 718-737. doi:10.2514/2.2824.

${ }^{25}$ Baruh, H., Analytical Dynamics, McGraw-Hill Higher Education, 1999. doi:ISBN 0073659770, 9780073659770, Chpt. 4.

${ }^{26}$ Caetano, J. V., Verboom, J., de Visser, C., de Croon, G., Remes, B., de Wagter, C., and Mulder, M., "Near-Hover Flapping Wing MAV Aerodynamic Modelling - a linear model approach," International Conference and Competition on Micro Air Vehicles, IMAV, 2013.

${ }^{27}$ Caetano, J. V., de Visser, C. C., Remes, B., de Wagter, C., and Mulder, M., "Controlled flight maneuvers of a Flapping Wing Micro Air Vehicle: a step towards the DelFly II identification," $A I A A$ Atmospheric Flight Mechanics Conference, No. 2013-4843, AIAA, 2013. doi 10.2514/6.2013-4843.

${ }^{28}$ Caetano, J. V., de Visser, C. C., Remes, B., de Wagter, C., and Mulder, M., "Modeling a Flapping Wing MAV: Flight Path Reconstruction of the DelFly II," AIAA Modeling and Simulation Technologies, No. 2013-4597, 2013. doi:10.2514/6.2013-4597.

${ }^{29}$ Lim, G., Shkarayev, S., Goff, Z., and Beran, P., "Studies of Flight Kinematics of Ornithopters," International Competition and Conference on Micro Air Vehicles, IMAV 2012, IMAV 2012, 2012.

${ }^{30}$ Shkarayev, S. and Maniar, G., "Experimental and computational modeling of the kinematics and aerodynamics of membrane flapping wings," 50th AIAA Aerospace Sciences Meeting including the New Horizons Forum and Aerospace Exposition, No. 2012-1208, AIAA, Nasville, Tennessee, January 2012.

${ }^{31}$ Hsiao, F. Y., Yang, T. M., and Lu, W. C., "Dynamics of Flapping-Wing MAVs: Application to the Tamkang Golden Snitch," Journal of Applied Science and Engineering, Vol. 15, No. 3, 2012, pp. $227-238$. 